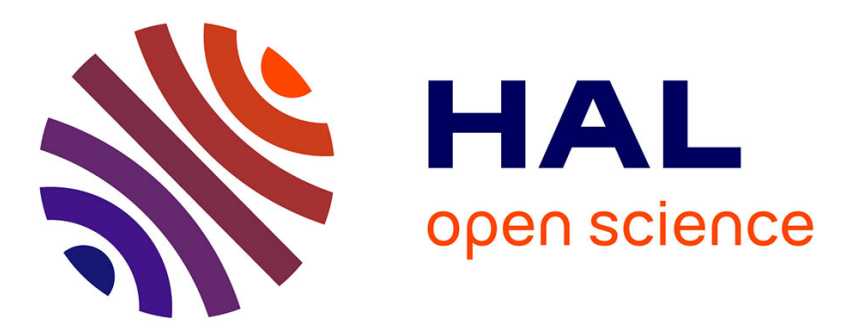

\title{
Subspace inverse power method and Polynomial Chaos representation for the modal frequency responses of random mechanical systems
}

\author{
Emmanuel Pagnacco, E Souza de Cursi, R Sampaio
}

\section{- To cite this version:}

Emmanuel Pagnacco, E Souza de Cursi, R Sampaio. Subspace inverse power method and Polynomial Chaos representation for the modal frequency responses of random mechanical systems. Computational Mechanics, 2016. hal-03449787

\section{HAL Id: hal-03449787 \\ https://hal.science/hal-03449787}

Submitted on 25 Nov 2021

HAL is a multi-disciplinary open access archive for the deposit and dissemination of scientific research documents, whether they are published or not. The documents may come from teaching and research institutions in France or abroad, or from public or private research centers.
L'archive ouverte pluridisciplinaire HAL, est destinée au dépôt et à la diffusion de documents scientifiques de niveau recherche, publiés ou non, émanant des établissements d'enseignement et de recherche français ou étrangers, des laboratoires publics ou privés. 


\title{
Subspace inverse power method and Polynomial Chaos representation for the modal frequency responses of random mechanical systems
}

\author{
E. Pagnacco*, E. Souza de Cursi* \& R. Sampaio^
}

20 January 2016

* INSA-Rouen, BP8, 76801 St.-Etienne du Rouvray Cedex, France

^ PUC-Rio, Mechanical Eng. Dept., Rua Marques de Sao Vicente, 225 22451-900 Rio de Janeiro, RJ Brazil

\begin{abstract}
This study concerns the computation of frequency responses of linear stochastic mechanical systems through a modal analysis. A new strategy, based on transposing standards deterministic deflated and subspace inverse power methods into stochastic framework, is introduced via polynomial chaos representation. Applicability and effectiveness of the proposed schemes is demonstrated through three simple application examples and one realistic application example. It is shown that null and repeated-eigenvalue situations are addressed successfully.
\end{abstract}

Keywords: Dynamic of structure, Frequency Response Function, Modal analysis, Propagation of uncertainty, Polynomial chaos, Clustered Eigenvalues, Rigid body modes

\section{Introduction}

This study concerns the propagation of uncertainties for structural dynamic problems. Over the past decades, methods for solving stochastic eigenproblems or dynamics problems have been predominantly based on either Monte Carlo Simulation (MCS) or perturbation methods such as Neumann or improved perturbations 
methods $[1,2,3,4,5,6]$. Implementation of MCS procedures is in principle straightforward while perturbation methods provide estimates of firsts moments, based on sensitivity analysis using Taylor series expansion of the quantity of interest. But these methods have several drawbacks. MCS is expensive in computing resources for large or complex problems or problems relying on several random variables, and perturbation methods have a limited radius of convergence for the involved series. Hence, efforts are constantly made to explore the suitability of spectral methods such as the Stochastic Reduced Basis Method (SRBM) [7, 8] and the Polynomial Chaos (PC) representations [4, 9, 10, 11]. Both of them pertain to a non-statistical approach to represent randomness. SRBM is based on the subspace spanned by the considered application. In [8], the practical basis suggested comes from a preconditioned stochastic Krylov subspace, performed from the nominal problem. Numerical studies for the first two moments on frequency response analysis of stochastic structural systems is addressed in this work, showing a degradation in results for large variations of the random variables. On the other hand, PC representation is a spectral representation of random processes which can be used in stochastic equations to represent unknown stochastic quantities [12, 13, 14, 11]. Since it uses a Hilbertian framework, the PC expansion is able to represent any second-order random quantity with any probability law.

In this work, efforts are pursued to explore the suitability of spectral methods. More specifically, stochastic solutions in the frequency domain are sought for linear problems when random properties are considered in the mechanical model in the inertial and elastic properties. Evaluating these random results are generally referred to as Stochastic Finite Element Methods (SFEM). Linear dynamic response is e.g. more efficiently dealt with in the modal space. To deal with the modal space one has first to solve an eigenvalue problem. This problem can either be a differential eigenvalue problem or a matrix eigenvalue problem, depending on whether a continuous model or a discrete model is used to describe the given system. For stochastic problems, probabilistic characterization of the eigensolutions of random matrix and of differential operators turn out to be of interest. SFEM with PC representation are well developed for linear algebraic systems, but are less developed for the random eigenvalue and for the random response of dynamic problems. The random eigenanalysis problem appears to be non linear and challenging conditions arise when part of the spectrum is clustered, that is, when there are repeated or closely spaced eigenvalues. In Ref. [4], the PC eigenvalue problem is approximated through a projection onto the deterministic normal mode basis, both for the normal mode equilibrium equation and for the normalization equation. In Ref. [9, 10], the eigenvalue problem with distinct roots is rewritten as a set of non-linear equations with a L2 normalization of the normal modes. Next, specific strategies are developed in Ref. [15, 16] to deal with repeated roots when such an approach 
is carried out. However, while the linear stochastic dynamic problem is carried out without approximation in this way -apart from the practical necessity of polynomial truncature-, the involved computational effort appears to be much more important than the one involved for static random problems. Hence, to be more efficient numerically, the Ref. [17] proposes to transpose in the random domain the deterministic inverse power method based on the spectral shift strategy. It appears to be a robust method to determine the random eigenvector associated to a random eigenvalue. But normalization of normal modes is achieved only in the mean sense in this work. This does not lead to favorable conditions to address next modal response of dynamic problems.

In this work, stochastic discrete linear systems, or discretized continuous systems, having -or not- null or repeated eigenvalues are considered for obtaining modal based frequency responses. PC representation through a weak characterization of mechanical equations is focused and efforts are made to solve the resulting stochastic modal equations and frequency responses without transforming this problem as it would be if, for example, projections onto the deterministic normal modes basis are involved. To achieve a numerical efficiency, the Newton-Raphson strategy is not selected and the deterministic eigenanalysis method such as the deflated inverse power method and the subspace inverse power method are transposed to the stochastic framework. The modal problem is addressed here without approximations on the normalization condition for the normal modes, by requiring that the stochastic normalization and the stochastic orthogonalization conditions must be satisfied. Then, modal based frequency responses take advantage of the stochastic modal mass-orthonormalization which is ensured. Moreover, for numerical efficiency, it is also a main objective of this work to keep the computational costs similar to the one required for the -well known and used- stochastic static problem solved by the PC method. Indeed, one numerical drawback of the intrusive PC strategy is the use of expectations of multiple PC products which requires both lot of operations to be computed and lot of memory to be stored. This aspect is a part of the curse of dimensionality problem which arises for stochastic modal based frequency responses analysis and it must keep in mind, as we will see in the following of this paper that expectations of a five PC products could be required to address these problems (while only expectations of a three PC products are required to address stochastic static problems). This problem can occur both for ensuring orthonormalization of the modal base and for obtaining modal based frequency responses. Hence, we will pay special attention to propose alternative numerical strategies at both these steps in order to use only expectations of PC products which are already used for stochastic static problems. Adoption of these propositions ensure the efficiency of the complete stochastic modal based frequency response analysis of this work by having computational costs similar to the one required for the stochastic static problem solved by 
the PC method.

The paper is organized as follows: the Sect. 2 addresses the modal dynamic problem, considering the deterministic and the stochastic frameworks. The Sect. 3 recalls basic considerations of the PC representation and uncertainties propagation in the context of the dynamic responses, either from the direct or from the modal way. The Sect. 4 addresses the proposed numerical strategies for the inverse power method and its deflated and subspace variants, by transposing the deterministic strategies to the stochastic ones. Then, four application examples are chosen to demonstrate the applicability and effectiveness of the proposed methodologies in Sect. 5. Applications to eigenanalysis random problems as well as frequency modal random responses are carried out for three simple application examples and one realistic application example. It is then demonstrated that the proposed strategies are applicable and that the proposed method is able to deal with situations for which part of the spectrum is clustered. Finally, a summary is given in Sect. 6 to conclude this work.

\section{Problem statement}

\subsection{Deterministic problem}

Let us considers the discrete -or discretized- linear deterministic problem of computing the dynamic response of a system subjected to a forcing which is defined in the frequency domain $(\omega=2 \pi f)$ :

$$
\left(\mathbf{k}-\omega^{2} \mathbf{m}+\mathbf{j} \mathbf{c} \omega\right) \mathbf{u}(\omega)=\mathbf{f}(\omega)
$$

where upright bold letters denote structural deterministic matrices. In this expression $\mathbf{k}, \mathbf{m}$, and $\mathbf{c}$ are $n$ dimensional real symmetric structural matrices, being respectively the stiffness, mass, and damping matrices. The frequency response functions are $\mathbf{u}(\omega)$ when $\mathbf{f}(\omega)$ is chosen as the $n \times n$ identity matrix $\mathbf{i}$ over a specified frequency band, $\omega_{\min } \leq \omega \leq \omega_{\max }$.

Since the problem is linear with respect to the frequency response, it is interesting to introduce the modal representation associated to the above spatial representation. Restricting ourselves to proportional-damped systems, the modal representation is real and is defined as:

$$
\left\{\begin{array}{c}
\mathrm{k} \phi=\mathrm{m} \phi \boldsymbol{\lambda} \\
\phi^{\mathrm{T}} \mathrm{m} \phi=\mu
\end{array}\right.
$$


with $\xi=\boldsymbol{\phi}^{\mathrm{T}} \mathbf{c} \boldsymbol{\phi}$ the modal damp matrix and where:

- $\boldsymbol{\phi}$ is the normal-mode matrix formed with the eigenvectors as columns;

- $\lambda$ is the diagonal matrix formed with the corresponding eigenvalues, that are the squares of the natural circular frequencies; and

- $\boldsymbol{\mu}$ is the diagonal modal mass matrix that is imposed to be the identity matrix, i.e. $\boldsymbol{\mu}=\mathbf{i}$, in this study.

Notice that a widely used convention consists in ordering the eigenvalues in ascending values, that is $\lambda_{1} \leq$ $\lambda_{2} \leq \cdots \leq \lambda_{n}$, while the associated eigenvectors are:

$$
\phi=\left\{\phi_{1}, \phi_{2}, \cdots, \phi_{n}\right\}
$$

Hence, when there is clustered eigenvalues, switching of the ordering of eigenvectors can occur for a small change in the physical parameters. For example, considering distinct natural frequencies of a simple plate with varying dimensions, ordering of a torsional and a flexural mode can be switched due to the modification of these frequencies. However, these two modes can be recognized experimentally, when compared before and after modifications, because they keep their shapes. Thus, a Modal Assurance Criterion (MAC) will indicate that they are nearly parallel, showing then that they correspond to the same physical modes ${ }^{1}$.

Then, the solution $\mathbf{u}(\omega)$ is such that:

$$
\mathbf{u}(\omega)=\boldsymbol{\phi} \mathbf{x}(\omega)=\sum_{r=1}^{n} \boldsymbol{\phi}_{i} \mathbf{x}_{i}(\omega)
$$

for $\mathbf{x}(\omega)$ which satisfies the uncoupled modal equations:

$$
\left(\lambda-\omega^{2} \mathbf{i}+\mathbf{j} \xi \omega\right) \mathbf{x}(\omega)=\boldsymbol{\phi}^{\mathrm{T}} \mathbf{f}(\omega)
$$

However, for mechanical applications which involves many degrees of freedom, an approximate solution is generally sought practically by truncating the modal basis to a specified range where only the lowest eigenvalues separated from the rest of the spectrum are considered: $\lambda_{1} \leq \cdots \leq \lambda_{n_{r}}<\lambda_{n_{r}+1} \leq \cdots \lambda_{n}$. This leads us to rewritten Eq. 3 as:

$$
\mathbf{u}(\omega) \simeq \sum_{r=1}^{n_{r}} \boldsymbol{\phi}_{i} \mathbf{x}_{i}(\omega)=\boldsymbol{\phi} \mathbf{x}(\omega)
$$

\footnotetext{
${ }^{1}$ However, care must be taken when natural frequencies are repeated. In this situation any combination of associated normal modes leads to another also valid normal mode; that is the associated eigenspace has dimension bigger than one. In such case, shapes of both modes of the previous example looks like a mix of the two original modes. Then, a specific procedure must be involved to avoid difficulties in following their evolution.
} 
where $\boldsymbol{\phi}$ becomes now a $n \times n_{r}$ matrix, while modal matrices are adjusted in their sizes accordingly.

\subsection{Stochastic problem}

When the system has random parameters, its response becomes a stochastic process, $\mathbf{U}(\omega, \varpi)$, such that

$$
\left(\mathbf{K}(\varpi)-\omega^{2} \mathbf{M}(\varpi)+\mathbf{j} \mathbf{C}(\varpi) \omega\right) \mathbf{U}(\omega, \varpi)=\mathbf{f}(\omega)
$$

for all $\varpi \in \Omega$ where $(\Omega, \mathcal{A}$, Prob) is the probability space associated with the underlying physical experiments and where upper case upright letters denotes random variables corresponding to deterministic ones which are denote by the same letter in lower case. The stochastic eigenproblem associated to this model is:

$$
\left\{\begin{array}{l}
\mathbf{K} \Phi=\mathrm{M} \Phi \boldsymbol{\Lambda} \\
\boldsymbol{\Phi}^{\mathrm{T}} \mathbf{M} \Phi=\mu
\end{array}\right.
$$

where the argument $\varpi$ is dropped for brevity. At this point, one has to note that this modal problem is slightly different from the one of Ref. $[17,10,15,16]$ due to the requested mass-orthonormalization condition. Then, $\mathbf{U}(\omega)$ can be express as:

$$
\mathbf{U}(\omega)=\mathbf{\Phi} \mathbf{X}(\omega)
$$

for:

$$
\left(\boldsymbol{\Lambda}-\omega^{2} \mathbf{i}+\mathbf{j} \boldsymbol{\Xi} \omega\right) \mathbf{X}(\omega)=\boldsymbol{\Phi}^{\mathrm{T}} \mathbf{f}(\omega)
$$

where:

$$
\boldsymbol{\Lambda}=\boldsymbol{\Phi}^{\mathrm{T}} \mathbf{K} \boldsymbol{\Phi} \quad \text { and } \quad \boldsymbol{\Xi}=\boldsymbol{\Phi}^{\mathrm{T}} \mathbf{C} \boldsymbol{\Phi} .
$$

One can notice that, due to the possible switching, or even mixing, of eigenvectors in the deterministic problem, the stochastic eigenproblem can be difficult to solve when there is clustered eigenvalues [16].

\section{Propagation of uncertainties using polynomial chaos representa- tion}

Several methods can be applied to get a representation of $\mathbf{U}(\omega)$ for given distributions of the random input properties $[6,11]$. From them, as an attractive alternative to the MCS, the PC representation consists in the 
expansion of the random process over a set of orthogonal polynomials. Wiener first introduced it in 1938 [12] and used only Hermite polynomial basis to represent Gaussian random processes. More recently, works on generalized Polynomial Chaos propose a list of polynomial basis corresponding to non-Gaussian distributions [18]. In effect, one can use any distribution as kernel and orthogonalize a polynomial basis of $L_{2}$ with respect to the probability measure generated by the chosen distribution. [19] demonstrated the $L_{2}$-convergence for any $L_{2}$ functionals, that is for any second-order random process (i.e. with finite variance).

\subsection{Direct dynamic frequency response}

Considering a generic random vector or random matrix, $\mathbf{G}$, the $\mathrm{PC}$ representation proposes to express it as a polynomial (truncation of a series) using a set of $n_{g}+1$ orthogonal polynomials, denoted $\psi_{g}$, in variables $\zeta_{i}, i \in\{1,, 2, \ldots d\}$, that are collected in a $d$-dimensional vector $\zeta$ :

$$
\mathbf{G}(\varpi)=\sum_{g=0}^{n_{g}} \boldsymbol{\Psi}_{g}(\boldsymbol{\zeta}(\varpi)) \boldsymbol{g}_{g}
$$

where $n_{g}+1=\frac{(d+h) !}{d ! h !}, h$ being the order of the representation. Notice that italic bold letters are used for vectors of the spectral representation. Generally, for a practical implementation, the order of expansion results from a truncation which has to be chosen accordingly a suitable criterion. For the structural matrices $\mathbf{K}(\boldsymbol{\zeta}), \mathbf{M}(\boldsymbol{\zeta})$, or $\mathbf{C}(\boldsymbol{\zeta})$ and for responses $\mathbf{U}(\omega, \boldsymbol{\zeta})$ the adopted PC representation are:

$$
\begin{aligned}
& \mathbf{K}(\boldsymbol{\zeta})=\sum_{k=0}^{n_{k}} \boldsymbol{\Psi}_{k}(\boldsymbol{\zeta}) \boldsymbol{k}_{k} \\
& \mathbf{M}(\boldsymbol{\zeta})=\sum_{\substack{n_{m}=0 \\
n_{c}}}^{\boldsymbol{\Psi}_{m}(\boldsymbol{\zeta}) \boldsymbol{m}_{m}} \\
& \mathbf{C}(\boldsymbol{\zeta})=\sum_{c=0}^{\boldsymbol{\Psi}_{c}(\boldsymbol{\zeta}) \boldsymbol{c}_{c}}
\end{aligned}
$$

and:

$$
\mathbf{U}(\omega, \boldsymbol{\zeta})=\sum_{u=0}^{n_{u}} \boldsymbol{\Psi}_{u} \boldsymbol{u}_{u}(\omega)
$$

Coming back to the generic random vector or matrix $\mathbf{G}$, the $n_{g}$ vectors or matrices of deterministic coefficients $\boldsymbol{g}_{g}$, now used to describe $\mathbf{G}$, can be evaluated in two ways: using an intrusive or a non-intrusive method. The non-intrusive method can be always used to find the coefficients of the stochastic variables when they are represented by a PC expansion. It uses the orthogonality of the polynomials with respect to the appropriated inner product to evaluate each vector of deterministic coefficients $\boldsymbol{g}_{g}$ : 


$$
\boldsymbol{g}_{g}=\frac{<\mathbf{G}(\boldsymbol{\zeta}), \boldsymbol{\Psi}_{g}(\boldsymbol{\zeta})>}{<\boldsymbol{\Psi}_{g}(\boldsymbol{\zeta}), \boldsymbol{\Psi}_{g}(\boldsymbol{\zeta})>}
$$

where $<\mathbf{G}(\boldsymbol{\zeta}), \boldsymbol{\Psi}_{g}(\boldsymbol{\zeta})>=\int_{\mathbb{R}^{d}} \mathbf{G}(\boldsymbol{\zeta}) \boldsymbol{\Psi}_{g}(\boldsymbol{\zeta}) p_{\boldsymbol{\zeta}}(\boldsymbol{\zeta}) \mathrm{d} \boldsymbol{\zeta}$ represents the inner product introduced by the PDF $p_{\boldsymbol{\zeta}}(\boldsymbol{\zeta})$. To evaluate this inner product, it is introduced a partition, $\boldsymbol{\zeta}_{i}$, the integrations points, $\mathbf{w}$ the vector which collects the quadrature weights and $n_{\mathrm{gp}}$ the number of points, the above integration can be done using a Gauss quadrature rule ([20]):

$$
<\mathbf{G}(\boldsymbol{\zeta}), \boldsymbol{\Psi}_{g}(\boldsymbol{\zeta})>=\sum_{i=1}^{n_{\mathrm{GP}}} \mathbf{G}\left(\boldsymbol{\zeta}_{i}\right) \boldsymbol{\Psi}_{g}\left(\boldsymbol{\zeta}_{i}\right)\{\mathbf{w}\}_{i}
$$

In this way, the residual associated to the difference between $\mathbf{G}(\varpi)$ and its PC representation is orthogonal to its PC representation. This strategy to determine the representation can be referred to as a strong characterization [16] and can be used to find coefficients of input stochastic variables such as $\boldsymbol{k}_{k}, \boldsymbol{m}_{m}$ or $\boldsymbol{c}_{c}$ for the structural matrices as well as to model the output stochastic variables such as $\boldsymbol{u}_{u}(\omega)$ for responses $\mathbf{U}(\omega, \zeta)$.

The intrusive method, or weak characterization, follows a variational approach. As a first step, the PC representation introduced for the stochastic variables are put into the governing equations. For instance, Eq. (4) produces:

$$
\begin{array}{r}
\left(\sum_{k=0}^{n_{k}} \boldsymbol{\Psi}_{k} \boldsymbol{k}_{k}-\omega^{2} \sum_{m=0}^{n_{m}} \boldsymbol{\Psi}_{m} \boldsymbol{m}_{m}+\mathrm{j} \omega \sum_{c=0}^{n_{c}} \boldsymbol{\Psi}_{c} \boldsymbol{c}_{c}\right)\left(\sum_{u=0}^{n_{u}} \boldsymbol{\Psi}_{u} \boldsymbol{u}_{u}(\omega)\right) \\
=\mathbf{f}(\omega) .
\end{array}
$$

Next, the vectors coefficients are given by solving the system which is obtained by taking expectations of the equations produced by the projection of Eq. (8) onto the set of polynomials $\Psi$ :

$$
\begin{array}{r}
\sum_{u=0}^{n_{u}}\left(\sum_{k=0}^{n_{k}}\left\langle\boldsymbol{\Psi}_{i} \boldsymbol{\Psi}_{k} \boldsymbol{\Psi}_{u}\right\rangle \boldsymbol{k}_{k}-\omega^{2} \sum_{m=0}^{n_{m}}\left\langle\boldsymbol{\Psi}_{i} \boldsymbol{\Psi}_{m} \boldsymbol{\Psi}_{u}\right\rangle \boldsymbol{m}_{m}+\right. \\
\left.\mathrm{j} \omega \sum_{c=0}^{n_{c}}\left\langle\boldsymbol{\Psi}_{i} \boldsymbol{\Psi}_{c} \boldsymbol{\Psi}_{u}\right\rangle \boldsymbol{c}_{c}\right) \boldsymbol{u}_{u}(\omega)=\left\langle\boldsymbol{\Psi}_{i}\right\rangle \mathbf{f}(\omega)
\end{array}
$$

for $i=0, \ldots, n_{u}$. This leads to a deterministic matricial system of the form:

$$
\left(\mathcal{K}-\omega^{2} \mathcal{M}+\mathrm{j} \mathcal{C} \omega\right) \mathcal{U}(\omega)=\mathcal{F}(\omega)
$$


where:

$$
\begin{aligned}
& \mathcal{K}=\left[\begin{array}{ccc}
\sum_{k=0}^{n_{k}}\left\langle\boldsymbol{\Psi}_{0} \boldsymbol{\Psi}_{k} \boldsymbol{\Psi}_{0}\right\rangle \boldsymbol{k}_{k} & \sum_{k=0}^{n_{k}}\left\langle\boldsymbol{\Psi}_{0} \boldsymbol{\Psi}_{k} \boldsymbol{\Psi}_{1}\right\rangle \boldsymbol{k}_{k} & \ldots \\
\sum_{k=0}^{n_{k}}\left\langle\boldsymbol{\Psi}_{1} \boldsymbol{\Psi}_{k} \boldsymbol{\Psi}_{0}\right\rangle \boldsymbol{k}_{k} & \sum_{k=0}^{n_{k}}\left\langle\boldsymbol{\Psi}_{1} \boldsymbol{\Psi}_{k} \boldsymbol{\Psi}_{1}\right\rangle \boldsymbol{k}_{k} & \\
\vdots & & \ddots
\end{array}\right], \\
& \mathcal{U}=\left[\begin{array}{c}
\boldsymbol{u}_{0} \\
\boldsymbol{u}_{1} \\
\vdots
\end{array}\right] \text { and } \quad \mathcal{F}=\left[\begin{array}{c}
\left\langle\boldsymbol{\Psi}_{0}\right\rangle \mathbf{f} \\
\left\langle\boldsymbol{\Psi}_{1}\right\rangle \mathbf{f} \\
\vdots
\end{array}\right]
\end{aligned}
$$

where calligraphic letters denote the structural PC representation matrices used for the analysis. The matrices $\mathcal{M}$ and $C$ have similar form as the matrix $\mathcal{K}$. Equation (10) is a linear system of deterministic equations which involves expectations of three PC products.

From a numerical point of view, the weak characterization provides a set of $n \times n_{g}$ coupled algebraic equations, while the strong characterization determines $\boldsymbol{g}_{g}$ from integrations, requiring at least a set of experiments for a quadrature rule. When the order of representation is adequate, the coefficients obtained from both characterizations should be identical when the same orthogonal basis is considered. However, this is not true for an inadequate truncation since the weak characterization couples the coefficients, while the strong characterization determines each coefficient independently.

\subsection{Modal frequency response}

The direct strategy proposed in the previous section by using the intrusive method is expensive numerically when considering a large frequency band since it involves solving a large system many times. As the structural matrices do not depend on the frequency, the modal response presented in subsection 2.2 is relevant. Considering only a particular, single mode, $\boldsymbol{\Phi}_{r}$, modal quantities can be represented in the chosen basis as:

$$
\begin{aligned}
\boldsymbol{\Phi}_{r}(\boldsymbol{\zeta}) & =\sum_{\phi=0}^{n_{\lambda}} \boldsymbol{\Psi}_{\phi}(\boldsymbol{\zeta}) \phi_{r \phi}, \\
\boldsymbol{\Lambda}_{r}(\boldsymbol{\zeta}) & =\sum_{\lambda=0}^{n_{\lambda}} \boldsymbol{\Psi}_{\lambda}(\boldsymbol{\zeta}) \boldsymbol{\lambda}_{r \lambda}
\end{aligned}
$$


and the Eq. (5), taken in the weak sense, becomes:

$$
\left\{\begin{array}{c}
\sum_{\phi=0}^{n_{\lambda}} \sum_{k=0}^{n_{k}}\left\langle\boldsymbol{\Psi}_{i} \boldsymbol{\Psi}_{k} \boldsymbol{\Psi}_{\phi}\right\rangle \boldsymbol{k}_{k} \boldsymbol{\phi}_{r \phi}= \\
\sum_{\phi=0}^{n_{\lambda}} \sum_{\lambda=0}^{n_{\lambda}} \sum_{m=0}^{n_{m}}\left\langle\boldsymbol{\Psi}_{i} \boldsymbol{\Psi}_{m} \boldsymbol{\Psi}_{\phi} \boldsymbol{\Psi}_{\lambda}\right\rangle \boldsymbol{m}_{m} \boldsymbol{\phi}_{r \phi} \boldsymbol{\lambda}_{r \lambda} \\
\sum_{\phi=0}^{n_{\lambda}} \sum_{m=0}^{n_{m}} \sum_{\lambda=0}^{n_{\lambda}}\left\langle\boldsymbol{\Psi}_{i} \boldsymbol{\Psi}_{\phi} \boldsymbol{\Psi}_{m} \boldsymbol{\Psi}_{\lambda}\right\rangle \boldsymbol{\phi}_{r \phi}^{\mathrm{T}} \boldsymbol{m}_{m} \boldsymbol{\phi}_{r \lambda}=\left\langle\boldsymbol{\Psi}_{i}\right\rangle \boldsymbol{\mu}
\end{array}\right.
$$

for $i=0, \ldots, n_{\lambda}$ which can be rewritten in a matricial form as:

$$
\left\{\begin{array}{l}
\mathcal{K} \Phi_{r}=\mathcal{M}^{\prime} \Phi_{r} \Lambda_{r} \\
\Phi_{r}^{\mathrm{T}} \mathcal{M}^{\prime} \Phi_{r}=\Upsilon
\end{array}\right.
$$

when collecting:

$$
\begin{aligned}
& \Phi_{r}=\left[\begin{array}{c}
\phi_{r 0} \\
\phi_{r 1} \\
\vdots
\end{array}\right], \quad \Lambda_{r}=\left[\begin{array}{c}
\lambda_{r 0} \\
\boldsymbol{\lambda}_{r 1} \\
\vdots
\end{array}\right], \\
& \Upsilon=\left[\begin{array}{c}
\left\langle\boldsymbol{\Psi}_{0}\right\rangle \boldsymbol{\mu} \\
\left\langle\boldsymbol{\Psi}_{1}\right\rangle \boldsymbol{\mu} \\
\vdots
\end{array}\right]=\left[\begin{array}{c}
\left\langle\boldsymbol{\Psi}_{0}\right\rangle \mathbf{i} \\
\mathbf{0} \\
\vdots
\end{array}\right]
\end{aligned}
$$

and:

$$
\begin{aligned}
& \mathcal{M}^{\prime} \Phi_{r}=
\end{aligned}
$$

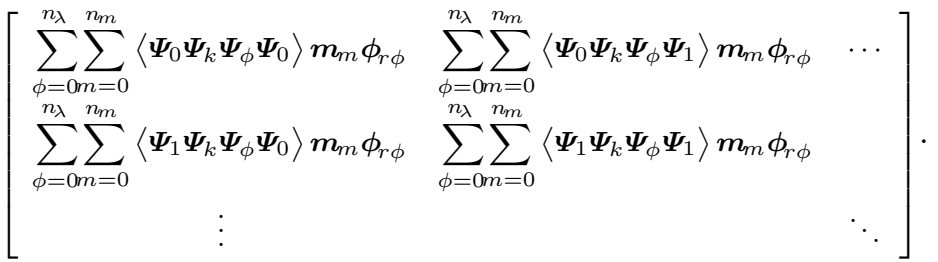

This is a non-linear system of deterministic equations which involves expectations of four PC products. The reference [10] proposes to solve it using a Newton-Raphson method starting from a statistically sampled initial value.

Then, synthesis of $\mathbf{U}(\omega)$ leads to consider:

$$
\mathbf{U}(\omega)=\sum_{r=1}^{n_{r}} \sum_{\phi=0}^{n_{\lambda}} \boldsymbol{\Psi}_{\phi} \phi_{r \phi} \mathbf{X}_{r}(\omega)
$$


for $\mathbf{X}_{r}(\omega)=\sum_{x=0}^{n_{x}} \boldsymbol{\Psi}_{x} \boldsymbol{x}_{r x}(\omega)$ such that:

$$
\left(\boldsymbol{\Phi}_{r}^{\mathrm{T}} \mathbf{K} \boldsymbol{\Phi}_{r}-\omega^{2} \boldsymbol{\Phi}_{r}^{\mathrm{T}} \mathbf{M} \boldsymbol{\Phi}_{r}+\mathrm{j} \boldsymbol{\Phi}_{r}^{\mathrm{T}} \mathbf{C}_{r} \boldsymbol{\Phi}_{r} \omega\right) \mathbf{X}_{r}(\omega)=\boldsymbol{\Phi}_{r}^{\mathrm{T}} \mathbf{f}(\omega)
$$

or:

$$
\left(\boldsymbol{\Phi}_{r}^{\mathrm{T}} \mathbf{K} \boldsymbol{\Phi}_{r}-\omega^{2}+\mathrm{j} \boldsymbol{\Phi}_{r}^{\mathrm{T}} \mathbf{C} \boldsymbol{\Phi}_{r} \omega\right) \mathbf{X}_{r}(\omega)=\boldsymbol{\Phi}_{r}^{\mathrm{T}} \mathbf{f}(\omega)
$$

since normal modes are mass normalized. It is, in the weak sense:

$$
\begin{array}{r}
\sum_{x=0}^{n_{x}}\left(\sum_{\phi=0}^{n_{\lambda}} \sum_{k=0}^{n_{k}} \sum_{\lambda=0}^{n_{\lambda}}\left\langle\boldsymbol{\Psi}_{i} \boldsymbol{\Psi}_{\phi} \boldsymbol{\Psi}_{k} \boldsymbol{\Psi}_{\lambda} \boldsymbol{\Psi}_{x}\right\rangle \boldsymbol{\phi}_{r \phi}^{\mathrm{T}}\left(\boldsymbol{k}_{k}+\mathrm{j} \omega \boldsymbol{c}_{k}\right) \boldsymbol{\phi}_{r \lambda}\right. \\
\left.-\omega^{2}\left\langle\boldsymbol{\Psi}_{i} \boldsymbol{\Psi}_{x}\right\rangle\right) \boldsymbol{x}_{r x}(\omega)=\left\langle\boldsymbol{\Psi}_{i}^{2}\right\rangle \boldsymbol{\phi}_{r i}^{\mathrm{T}} \mathbf{f}(\omega)
\end{array}
$$

for $i=0, \ldots, n_{x}$. This is a linear system of deterministic equations which involves expectations of five PC products. Then it is more efficient computationally to use PC representation of modal quantities. Using $\boldsymbol{\Lambda}_{r}=\sum_{\lambda=0}^{n_{\lambda}} \boldsymbol{\Psi}_{\lambda} \boldsymbol{\lambda}_{r \lambda}$ and $\boldsymbol{\Xi}_{r}=\sum_{\xi=0}^{n_{\xi}} \boldsymbol{\Psi}_{\xi} \boldsymbol{\xi}_{r \xi}$, the above expression is rewritten:

$$
\left(\boldsymbol{\Lambda}_{r}-\omega^{2}+\mathbf{j} \Xi_{r} \omega\right) \mathbf{X}_{r}(\omega)=\mathbf{\Phi}_{r}^{\mathrm{T}} \mathbf{f}(\omega)
$$

to produce, in the weak sense:

$$
\begin{aligned}
\sum_{x=0}^{n_{x}}\left(\sum_{\lambda=0}^{n_{\lambda}}\left\langle\boldsymbol{\Psi}_{i} \boldsymbol{\Psi}_{\lambda} \boldsymbol{\Psi}_{x}\right\rangle \boldsymbol{\lambda}_{r \lambda}-\omega^{2}\left\langle\boldsymbol{\Psi}_{i} \boldsymbol{\Psi}_{x}\right\rangle\right. \\
\left.\quad+\mathrm{j} \omega \sum_{\xi=0}^{n_{\xi}}\left\langle\boldsymbol{\Psi}_{i} \boldsymbol{\Psi}_{\xi} \boldsymbol{\Psi}_{x}\right\rangle \boldsymbol{\xi}_{r \xi}\right) \boldsymbol{x}_{r x}(\omega)=\left\langle\boldsymbol{\Psi}_{i}^{2}\right\rangle \phi_{r i}^{\mathrm{T}} \mathbf{f}(\omega)
\end{aligned}
$$

for $i=0, \ldots, n_{x}$. It is a linear system of deterministic equations which involves expectations of three PC products. This leads to a deterministic matricial system of the form:

$$
\left(\mathcal{J}_{r}-\omega^{2} \mathcal{I}_{r}+\mathrm{j} \mathcal{D}_{r} \omega\right) \mathcal{X}_{r}(\omega)=\mathcal{R}_{r}(\omega)
$$


where:

$$
\begin{aligned}
& \mathcal{J}_{r}=\left[\begin{array}{ccc}
\sum_{\lambda=0}^{n_{\lambda}}\left\langle\boldsymbol{\Psi}_{0} \boldsymbol{\Psi}_{\lambda} \boldsymbol{\Psi}_{0}\right\rangle \boldsymbol{\lambda}_{r \lambda} & \sum_{\lambda=0}^{n_{\lambda}}\left\langle\boldsymbol{\Psi}_{0} \boldsymbol{\Psi}_{\lambda} \boldsymbol{\Psi}_{1}\right\rangle \boldsymbol{\lambda}_{r \lambda} & \cdots \\
\sum_{\lambda=0}^{n_{\lambda}}\left\langle\boldsymbol{\Psi}_{1} \boldsymbol{\Psi}_{\lambda} \boldsymbol{\Psi}_{0}\right\rangle \boldsymbol{\lambda}_{r \lambda} & \sum_{\lambda=0}^{n_{\lambda}}\left\langle\boldsymbol{\Psi}_{1} \boldsymbol{\Psi}_{\lambda} \boldsymbol{\Psi}_{1}\right\rangle \boldsymbol{\lambda}_{r \lambda} & \\
\vdots & & \ddots
\end{array}\right], \\
& \mathcal{X}_{r}=\left[\begin{array}{c}
x_{r 0} \\
x_{r 1} \\
\vdots
\end{array}\right] \quad \text { and } \quad \mathcal{R}_{r}=\left[\begin{array}{c}
\left\langle\boldsymbol{\Psi}_{0}^{2}\right\rangle \boldsymbol{\phi}_{r 0}^{\mathrm{T}} \mathbf{f} \\
\left\langle\boldsymbol{\Psi}_{1}^{2}\right\rangle \boldsymbol{\phi}_{r 1}^{\mathrm{T}} \mathbf{f} \\
\vdots
\end{array}\right],
\end{aligned}
$$

while matrices $\mathcal{I}_{r}$ and $D_{r}$ have a similar form to the matrix $\mathcal{J}_{r}$.

Thus, to evaluate $\mathbf{U}(\omega)$ through Eq. (13) once the stochastic eigenproblem is solved, assuming it provides the PC representation of eigenvectors and eigenvalues, it is efficient to solve Eq. (15) to obtain the modal displacement matrix coefficients (that is a vector, if $\mathbf{f}(\omega)$ also is). It corresponds to the equation of a single degree of freedom system, thanks to the normal modes basis that decouples mechanical degrees of freedoms. This is the intrusive strategy which is chosen in this work to solve the stochastic dynamic problem by the modal strategy. But it remains necessary to propose an efficient numerical strategy to solve the system (12) which defines the normal modes base. This is the subject of the next section.

\section{Numerical methods for the determination of normal modes bases}

Considering the deterministic generalized normal modes basis problem, it exists numerical methods dedicated to its efficient determination [21,22]. Let us first describe the ones we propose to transpose in the random domain.

\subsection{Deterministic methods}

From a numerical point of view, Lanczos method and power method are adapted to find eigenvectors of large numerical problems. The inverse power method -or inverse iterations method- is the most basic method of computing an eigenvector $\boldsymbol{\phi}_{1}$ associated to the lowest eigenvalue $\left|\boldsymbol{\lambda}_{1}\right|>0$ for the generalized problem (2) when considering a positive-definite symmetric and real stiffness matrix. The basic algorithm of this method is:

1. Choose an initial vector $\boldsymbol{\phi}_{1}^{(0)}$ such that $\boldsymbol{\phi}_{1}^{(0) \mathrm{T}} \mathbf{m} \boldsymbol{\phi}_{1}^{(0)}=1$,

2. For $k=1,2, \ldots$ do: 
(a) Evaluate $\mathbf{f}_{1}^{(k)}=\mathbf{m} \phi_{1}^{(k-1)}$

(b) Solve $\mathbf{k} \boldsymbol{\phi}_{1}^{(k)}=\mathbf{f}_{1}^{(k)}$

(c) Normalize $\boldsymbol{\phi}_{1}^{(k)}$ such that $\boldsymbol{\phi}_{1}^{(k) \mathrm{T}} \mathbf{m} \boldsymbol{\phi}_{1}^{(k)}=1$

3. Let $\boldsymbol{\phi}_{1} \leftarrow \boldsymbol{\phi}_{1}^{(k)}$ and compute $\lambda_{1}=\boldsymbol{\phi}_{1}^{\mathrm{T}} \mathbf{k} \boldsymbol{\phi}_{1}$

4. End

This algorithm repeats the step 2 until $\boldsymbol{\phi}_{1}^{(k)}$ converges to within some tolerance $\epsilon$. A variant is obtained by including the step 3 in the loop of step 2, enabling to test the convergence on $\lambda_{1}^{(k)}$, being $\left|\lambda_{1}^{(k)}-\lambda_{1}^{(k-1)}\right|<$ $\epsilon\left|\lambda_{1}^{(k)}\right|$. Noticed that even if $\boldsymbol{\phi}_{1}^{(0)}$ is orthogonal to $\boldsymbol{\phi}_{1}$, it is expected that $\boldsymbol{\phi}_{1}$ would be recovered numerically due to round-off errors.

Then, deflation can be carried out to construct the next lowest eigenvalue $\lambda_{2}$ and so on for the next ones. In such a way, the eigenvalue $\boldsymbol{\lambda}_{r}$, for $r>1$, is obtained by introducing an orthogonalization step before the normalization, as:

$$
\boldsymbol{\phi}_{r}^{(k)}=\boldsymbol{\phi}_{r}^{(k)}-\sum_{q=1}^{r-1} \boldsymbol{\phi}_{q} \boldsymbol{\phi}_{q}^{\mathrm{T}} \mathbf{m} \boldsymbol{\phi}_{r}^{(k)}
$$

This orthogonalization step is also necessary when considering semi-definite stiffness matrix where the first $n_{0}$ eigenvectors $\boldsymbol{\phi}_{\mathrm{RBM}}$ correspond to the null space of $\mathbf{k}$, being $\mathbf{k} \boldsymbol{\phi}_{\mathrm{RBM}}=\mathbf{0}$. In such situation, a generalized inverse is applied within iterations. Then, for $n_{r}$ elastic modes, the adopted deflated inverse power method algorithm is:

1. Find the null space $\boldsymbol{\phi}_{\mathrm{RBM}}$ of $\mathbf{k}$ such that $\boldsymbol{\phi}_{\mathrm{RBM}}^{\mathrm{T}} \mathbf{m} \boldsymbol{\phi}_{\mathrm{RBM}}=\mathbf{i}_{0}$ and let $\boldsymbol{\phi} \leftarrow \boldsymbol{\phi}_{\mathrm{RBM}}$

2. For each sought mode $r=n_{0}+1, n_{0}+2, \ldots, n_{r}$, do:

(a) Choose an initial vector $\boldsymbol{\phi}_{r}^{(0)}$ such that

$$
\boldsymbol{\phi}_{\mathrm{RBM}}^{\mathrm{T}} \mathbf{m} \boldsymbol{\phi}_{r}^{(0)}=\mathbf{0} \text { and } \boldsymbol{\phi}_{r}^{(0) \mathrm{T}} \mathbf{m} \boldsymbol{\phi}_{r}^{(0)}=1,
$$

(b) Compute $\lambda_{r}^{(0)}=\boldsymbol{\phi}_{r}^{(0) \mathrm{T}} \mathbf{k} \boldsymbol{\phi}_{r}^{(0)}$

(c) For $k=1,2, \ldots$ do:

i. Evaluate $\mathbf{f}_{r}^{(k)}=\mathbf{m} \boldsymbol{\phi}_{r}^{(k-1)}$

ii. Solve $\mathbf{k} \boldsymbol{\phi}_{r}^{(k)}=\mathbf{f}_{r}^{(k)}$

iii. Orthonormalize $\boldsymbol{\phi}_{r}^{(k)}$ such that

$$
\boldsymbol{\phi}^{\mathrm{T}} \mathbf{m} \boldsymbol{\phi}_{r}^{(k)}=\mathbf{0} \text { and }\left\|\boldsymbol{\phi}_{r}^{(k)}\right\|_{\mathbf{m}}=1
$$


iv. Compute $\lambda_{r}^{(k)}=\boldsymbol{\phi}_{r}^{(k) \mathrm{T}} \mathbf{k} \boldsymbol{\phi}_{r}^{(k)}$

(d) Let $\boldsymbol{\phi} \leftarrow\left[\boldsymbol{\phi}, \boldsymbol{\phi}_{r}^{(k)}\right]$

3. End

where $\mathbf{i}_{0}$ is the $n_{0}$ identity matrix. However, this algorithm does not ensure to find the first eigenvectors when repeated eigenvalues exist. The inverse simultaneous vector iteration -or subspace inverse power methodhas not this drawback. It consists in handling simultaneously a set of modes $\boldsymbol{\phi}^{(k)}$, while preserving their orthonormality within iterations. Its basic algorithm is:

1. Find the null space $\boldsymbol{\phi}_{\mathrm{RBM}}$ of $\mathbf{k}$ such that $\boldsymbol{\phi}_{\mathrm{RBM}}^{\mathrm{T}} \mathbf{m} \boldsymbol{\phi}_{\mathrm{RBM}}=\mathbf{i}_{0}$

2. Choose an orthonormalized initial matrix $\boldsymbol{\phi}^{(0)}$ such that:

$\boldsymbol{\phi}_{\mathrm{RBM}}^{\mathrm{T}} \mathbf{m} \boldsymbol{\phi}^{(0)}=\mathbf{0}$ and $\boldsymbol{\phi}^{(0) \mathrm{T}} \mathbf{m} \boldsymbol{\phi}^{(0)}=\mathbf{i}_{r 0}$

3. Compute $\boldsymbol{\lambda}^{(0)}=\boldsymbol{\phi}^{(0) \mathrm{T}} \mathbf{k} \boldsymbol{\phi}^{(0)}$

4. For $k=1,2, \ldots$ do:

(a) Evaluate $\mathbf{f}^{(k)}=\mathbf{m} \boldsymbol{\phi}^{(k-1)}$

(b) Solve $\mathbf{k} \boldsymbol{\phi}^{(k)}=\mathbf{f}^{(k)}$

(c) Orthonormalize $\boldsymbol{\phi}^{(k)}$ such that:

$\boldsymbol{\phi}_{0}^{\mathrm{T}} \mathbf{m} \boldsymbol{\phi}^{(0)}=\mathbf{0}$ and $\boldsymbol{\phi}^{(k) \mathrm{T}} \mathbf{m} \boldsymbol{\phi}^{(k)}=\mathbf{i}_{r}$

(d) Compute $\boldsymbol{\lambda}^{(k)}=\boldsymbol{\phi}^{(k) \mathrm{T}} \mathbf{k} \boldsymbol{\phi}^{(k)}$

5. Let $\boldsymbol{\phi} \leftarrow\left[\boldsymbol{\phi}_{\mathrm{RBM}}, \boldsymbol{\phi}^{(k)}\right]$

6. End

where $\mathbf{i}_{r 0}$ is the $n_{r}-n_{0}$ identity matrix and $\boldsymbol{\phi}^{(0)}$ is the $n \times\left(n_{r}-n_{0}\right)$ matrix composed of starting guesses vectors. If it is not deficient in the directions of sought eigenvectors, convergence of this method is ensured for real symmetric semi-definite positive matrix $\mathbf{k}$.

\subsection{Determination of the stochastic normal modes basis using the deflated in- verse power method and polynomial chaos representation}

The deflated inverse power method is a suitable alternative to the Newton-Raphson method to solve the system of Eqs. (11) in order to build the stochastic normal mode basis. 
The proposed algorithm for the deflated inverse power method is the following:

1. Choose $\Phi_{\mathrm{RBM}}$ from null $(\mathbf{k})$ to represent $\boldsymbol{\Phi}_{\mathrm{RBM}}$

2. Normalize $\Phi_{\mathrm{RBM}}$ such that $\boldsymbol{\Phi}_{\mathrm{RBM}}^{\mathrm{T}} \mathbf{M} \boldsymbol{\Phi}_{\mathrm{RBM}}=\mathbf{i}$ and let $\Phi \leftarrow \Phi_{\mathrm{RBM}}$

3. For each sought mode $r$, do:

(a) From the deterministic vector $\phi_{r}$, define the initial vector $\Phi_{r}^{(0)}$ to represents $\boldsymbol{\Phi}_{r}^{(0)}$

(b) Find $\Phi_{r}^{(0) 1}$ having a unit modal mass from $\Phi_{r}^{(0)}$ such that $\boldsymbol{\Phi}^{\mathrm{T}} \mathbf{M} \Phi_{r}^{(k)}=\mathbf{0}$ and let $\Phi_{r}^{(0)} \leftarrow \Phi_{r}^{(0) 1}$

(c) Evaluate $\Lambda_{r}^{(0)}$ from a modal projection of $\mathbf{K}$ over $\boldsymbol{\Phi}_{r}^{(0)}$

(d) For $k=1,2, \ldots$ do:

i. Evaluate $\mathcal{F}_{r}^{(k)}=\mathcal{M} \Phi_{r}^{(k-1)}$

ii. Solve $\mathcal{K} \Phi_{r}^{(k)}=\mathcal{F}_{r}^{(k)}$

iii. Find $\Phi_{r}^{(k) \perp}$ that orthogonalizes $\boldsymbol{\Phi}_{r}^{(k)}$ to $\boldsymbol{\Phi}_{q}$ for $q \in\{1, \ldots, r-1\}$ and let $\Phi_{r}^{(k)} \leftarrow \Phi_{r}^{(k) \perp}$

iv. Evaluate $\Phi_{r}^{(k) 1}$ that normalizes modal mass to unity and let $\Phi_{r}^{(k)} \leftarrow \Phi_{r}^{(k) 1}$

v. Evaluate $\Lambda_{r}^{(k)}$ from a modal projection of $\mathbf{K}$ over $\Phi_{r}^{(k)}$

(e) Let $\Phi \leftarrow\left[\Phi, \Phi_{r}^{(k)}\right]$

\section{End}

In this algorithm, a simple and efficient starting guess for $\Phi_{r}^{(0)}$ is produced from the normalized normal mode $\phi_{r}$ of the deterministic nominal mechanical system, in which case $\boldsymbol{\Phi}_{r 0}^{(0)}=\phi_{r}$ while $\boldsymbol{\Phi}_{r \phi}^{(0)}=\mathbf{0}$ for $\phi>0$. Hence, for an efficient numerical scheme, it is recommended to systemically carried out the deterministic subspace inverse power method of the previous subsection 4.1 in order to produce efficiently all the starting guesses $\Phi_{r}^{(0)}$ for $r \in\left\{n_{r+1}, \ldots, n\right\}$. This choice ensure to find a meaningful set of first orthogonal eigenvectors, even when repeated eigenvalues exist for the nominal system. From our numerical experiment, it is found that this choice for the starting guess enables the algorithm to deal with deterministic nominal systems having repeated eigenfrequencies.

However, it has to be noted that even when the deterministic eigenvector is effectively mass normalized, its stochastic counterpart may not be mass normalized. Then, the normalization sub-step (b) of this algorithm holds in any cases. Modal projections, orthogonalization, and normalization steps are detailed in the following subsections. Notice that choosing the suggested starting guess for $\Phi_{r}^{(0)}$ enables the algorithm to deal with null eigenvalues, in which case the sub-step (d) is bypassed. 


\subsection{Normalization step}

The normalization step consists in searching $\boldsymbol{\Phi}_{r}^{(k) 1}$ which is defined by:

$$
\boldsymbol{\Phi}_{r}^{(k) 1 \mathrm{~T}} \mathbf{M} \boldsymbol{\Phi}_{r}^{(k) 1}=1
$$

It is achieved for:

$$
\boldsymbol{\Phi}_{r}^{(k) 1}=\frac{1}{\left\|\boldsymbol{\Phi}_{r}^{(k)}\right\|_{\mathbf{M}}} \boldsymbol{\Phi}_{r}^{(k)}
$$

where: $\quad\left\|\Phi_{r}^{(k)}\right\|_{\mathbf{M}} \quad=\quad\left(\boldsymbol{\Phi}_{r}^{(k) \mathrm{T}} \mathbf{M} \boldsymbol{\Phi}_{r}^{(k)}\right)^{1 / 2} \quad$ while $\boldsymbol{\Phi}_{r}^{(k) 1}(\boldsymbol{\zeta})=\sum_{\phi=0}^{n_{\lambda}} \boldsymbol{\Psi}_{\phi}(\boldsymbol{\zeta}) \phi_{r \phi}^{(k) 1}$ with $\phi_{r \phi}^{(k) 1}=\frac{<\boldsymbol{\Phi}_{r}^{(k) 1}, \boldsymbol{\Psi}_{\phi}(\boldsymbol{\zeta})>}{<\boldsymbol{\Psi}_{\phi}^{2}(\boldsymbol{\zeta})>}$.

In practice, it can be performed from a quadrature rule:

$$
\phi_{r \phi}^{(k) 1}=\frac{1}{<\boldsymbol{\Psi}_{\phi}^{2}(\boldsymbol{\zeta})>} \sum_{i=1}^{n_{\mathrm{gp}}} \boldsymbol{\Phi}_{r}^{(k) 1}\left(\boldsymbol{\zeta}_{i}\right) \boldsymbol{\Psi}_{\phi}\left(\boldsymbol{\zeta}_{i}\right) \mathbf{w}_{i}
$$

where:

$$
\boldsymbol{\Phi}_{r}^{(k) 1}\left(\zeta_{i}\right)=\left(\boldsymbol{\Phi}_{r}^{(k) \mathrm{T}}\left(\boldsymbol{\zeta}_{i}\right) \mathbf{M}\left(\boldsymbol{\zeta}_{i}\right) \boldsymbol{\Phi}_{r}^{(k)}\left(\boldsymbol{\zeta}_{i}\right)\right)^{-1 / 2} \boldsymbol{\Phi}_{r}^{(k)}\left(\boldsymbol{\zeta}_{i}\right)
$$

This is an enhanced formulation from a numerical point of view since:

- it does not involved the non-linear solution of the equations as the ones obtained by the weak characterization, $c f$. Eq. (11);

- it does not requires expectations of a four PC products as it is by the weak characterization.

\subsection{Orthogonalization step}

The orthogonalization step consists in searching the PC representation of $\boldsymbol{\Phi}_{r}^{(k) \perp}$ orthogonal to $\boldsymbol{\Phi}_{q}$, when knowing the PC representation of $\boldsymbol{\Phi}_{q}$ and the one of $\boldsymbol{\Phi}_{r}^{(k)}$ for $q<r$. For mass-normalized normal modes $\boldsymbol{\Phi}_{q}$, the orthogonalization condition is defined by:

$$
\boldsymbol{\Phi}_{q}^{\mathrm{T}} \mathbf{M} \boldsymbol{\Phi}_{r}^{(k) \perp}=0
$$

and it is achieved for:

$$
\boldsymbol{\Phi}_{r}^{(k) \perp}=\boldsymbol{\Phi}_{r}^{(k)}-\boldsymbol{\Phi}_{q} \boldsymbol{\Phi}_{q}^{\mathrm{T}} \mathbf{M} \boldsymbol{\Phi}_{r}^{(k)}
$$


which is for the $\mathrm{PC}$ representation of the stochastic variables:

$$
\begin{aligned}
& \sum_{\phi=0}^{n_{\lambda}} \boldsymbol{\Psi}_{\phi}(\boldsymbol{\zeta}) \boldsymbol{\phi}_{r \phi}^{(k) \perp}=\sum_{\phi=0}^{n_{\lambda}} \boldsymbol{\Psi}_{\phi}(\boldsymbol{\zeta}) \boldsymbol{\phi}_{r \phi}^{(k)} \\
& -\sum_{\phi=0}^{n_{\lambda}} \sum_{\varphi=0}^{n_{\lambda}} \sum_{m=0}^{n_{m}} \sum_{\lambda=0}^{n_{\lambda}} \boldsymbol{\Psi}_{\phi}(\boldsymbol{\zeta}) \boldsymbol{\Psi}_{\varphi}(\boldsymbol{\zeta}) \boldsymbol{\Psi}_{m}(\boldsymbol{\zeta}) \boldsymbol{\Psi}_{\lambda}(\boldsymbol{\zeta}) \phi_{q \phi} \boldsymbol{\phi}_{q \varphi}^{\mathrm{T}} \boldsymbol{m}_{m} \boldsymbol{\phi}_{r \lambda}^{(k)}
\end{aligned}
$$

Then, by taking:

$$
\begin{aligned}
& \phi_{r i}^{(k) \perp}=\phi_{r i}^{(k)} \\
& \quad-\frac{1}{\left\langle\boldsymbol{\Psi}_{i}^{2}\right\rangle} \sum_{\varphi=0}^{n_{\lambda}} \sum_{\phi=0}^{n_{\lambda}} \sum_{m=0}^{n_{m}} \sum_{\lambda=0}^{n_{\lambda}}\left\langle\boldsymbol{\Psi}_{i} \boldsymbol{\Psi}_{\phi} \boldsymbol{\Psi}_{\varphi} \boldsymbol{\Psi}_{m} \boldsymbol{\Psi}_{\lambda}\right\rangle \phi_{q \phi} \boldsymbol{\phi}_{q \varphi}^{\mathrm{T}} \boldsymbol{m}_{m} \boldsymbol{\phi}_{r \lambda}^{(k)}
\end{aligned}
$$

for $i=0, \ldots, n_{\lambda}$, it is ensured that:

$$
\begin{aligned}
& \sum_{\phi=0}^{n_{\lambda}}\left\langle\boldsymbol{\Psi}_{i} \boldsymbol{\Psi}_{\phi}\right\rangle \boldsymbol{\phi}_{r \phi}^{(k) \perp}=\sum_{\phi=0}^{n_{\lambda}}\left\langle\boldsymbol{\Psi}_{i} \boldsymbol{\Psi}_{\phi}\right\rangle \boldsymbol{\phi}_{r \phi}^{(k)} \\
& \quad-\sum_{\phi=0}^{n_{\lambda}} \sum_{\varphi=0}^{n_{\lambda}} \sum_{m=0}^{n_{m}} \sum_{\lambda=0}^{n_{\lambda}}\left\langle\boldsymbol{\Psi}_{i} \boldsymbol{\Psi}_{\phi} \boldsymbol{\Psi}_{\varphi} \boldsymbol{\Psi}_{m} \boldsymbol{\Psi}_{\lambda}\right\rangle \boldsymbol{\phi}_{q \phi} \boldsymbol{\phi}_{q \varphi}^{\mathrm{T}} \boldsymbol{m}_{m} \boldsymbol{\phi}_{r \lambda}^{(k)} .
\end{aligned}
$$

However, the above expression for $\phi_{r \phi}^{(k) \perp}$ involves expectations of five PC products. Thus, an enhanced more

efficient- strategy is desirable from a numerical point of view to find $\phi_{r \phi}^{(k) \perp}$. From:

$$
\sum_{\phi=0}^{n_{\lambda}} \boldsymbol{\Psi}_{\phi}\left(\boldsymbol{\zeta}_{i}\right) \phi_{r \phi}^{(k) \perp}=\boldsymbol{\Phi}_{r}^{(k) \perp}\left(\boldsymbol{\zeta}_{i}\right) \quad \forall i
$$

we know that:

$$
\phi_{r \phi}^{(k) \perp}=\frac{<\boldsymbol{\Phi}_{r}^{(k) \perp}, \boldsymbol{\Psi}_{\phi}(\boldsymbol{\zeta})>}{<\boldsymbol{\Psi}_{\phi}^{2}(\boldsymbol{\zeta})>}
$$

or:

$$
\phi_{r \phi}^{(k) \perp}=\frac{1}{<\boldsymbol{\Psi}_{\phi}^{2}(\boldsymbol{\zeta})>} \sum_{i=1}^{n_{\mathrm{gp}}}\left(\boldsymbol{\Psi}_{\phi}\left(\boldsymbol{\zeta}_{i}\right) \mathbf{w}_{i}\right)\left(\boldsymbol{\Psi}_{\phi}\left(\boldsymbol{\zeta}_{i}\right) \phi_{r \phi}^{(k) \perp}\right)
$$

when sampling points $\boldsymbol{\zeta}_{i}$ are the ones of the Gauss quadrature. To express $\left(\boldsymbol{\Psi}_{\phi}\left(\boldsymbol{\zeta}_{i}\right) \phi_{r \phi}^{(k) \perp}\right)$, it is known from the Eq. (16) that:

$$
\begin{aligned}
& \boldsymbol{\Phi}_{r}^{(k) \perp}(\boldsymbol{\zeta})=\sum_{\phi=0}^{n_{\lambda}} \boldsymbol{\Psi}_{\phi}(\boldsymbol{\zeta}) \phi_{r \phi}^{(k)} \\
& -\sum_{\phi=0 \rho=0}^{n_{\lambda}} \sum_{m=0}^{n_{\lambda}} \sum^{n_{m}} \sum_{\lambda}^{n_{\lambda}} \boldsymbol{\Psi}_{\phi}(\boldsymbol{\zeta}) \boldsymbol{\Psi}_{\varphi}(\boldsymbol{\zeta}) \boldsymbol{\Psi}_{m}(\boldsymbol{\zeta}) \boldsymbol{\Psi}_{\lambda}(\boldsymbol{\zeta}) \phi_{q \phi} \phi_{q \varphi}^{\mathrm{T}} \boldsymbol{m}_{m} \boldsymbol{\phi}_{r \lambda}^{(k)}
\end{aligned}
$$


and we state that the above equality holds if:

$$
\begin{aligned}
& \left(\boldsymbol{\Psi}_{\phi}\left(\boldsymbol{\zeta}_{j}\right) \phi_{r \phi}^{(k) \perp}\right)=\boldsymbol{\Psi}_{\phi}\left(\boldsymbol{\zeta}_{j}\right) \phi_{r \phi}^{(k)} \\
& -\sum_{\varphi=0 m=0 \lambda=0}^{n_{\lambda}} \sum_{m}^{n_{m}} \sum_{\boldsymbol{n}_{\lambda}}^{\boldsymbol{n}_{\phi}}\left(\boldsymbol{\zeta}_{j}\right) \boldsymbol{\Psi}_{\varphi}\left(\boldsymbol{\zeta}_{j}\right) \boldsymbol{\Psi}_{m}\left(\boldsymbol{\zeta}_{j}\right) \boldsymbol{\Psi}_{\lambda}\left(\boldsymbol{\zeta}_{j}\right) \phi_{q \phi} \boldsymbol{\phi}_{q \varphi}^{\mathrm{T}} \boldsymbol{m}_{m} \boldsymbol{\phi}_{r \lambda}^{(k)}
\end{aligned}
$$

for all $\zeta_{j}$. Hence, evaluating the above relation at sampling points $\zeta_{i}$ enables the evaluation of $\phi_{r \phi}^{(k) \perp}$ from the Eq. (17).

For large structural systems, this strategy is suitable to construct orthogonalized statics or inertial modes that are necessary to enrich a truncated normal mode basis, as well as for modal synthesis methods [21].

\subsection{Modal projections}

Modal projections of structural matrices are performed easily in a strong characterization. Considering a mass-normalized normal mode $\boldsymbol{\Phi}_{q}$ and the stiffness matrix $\mathbf{K}$, the modal projection $\boldsymbol{\Lambda}_{q}$ is defined as:

$$
\Lambda_{q}(\boldsymbol{\zeta})=\Phi_{q}^{\mathrm{T}}(\boldsymbol{\zeta}) \mathbf{K}(\boldsymbol{\zeta}) \boldsymbol{\Phi}_{q}(\boldsymbol{\zeta})
$$

while its PC representation is: $\boldsymbol{\Lambda}_{q}(\boldsymbol{\zeta})=\sum_{\lambda=0}^{n_{\lambda}} \boldsymbol{\Psi}_{\lambda}(\boldsymbol{\zeta}) \boldsymbol{\lambda}_{q \lambda}$ for: $\lambda_{q \lambda}=\frac{\left\langle\boldsymbol{\Lambda}_{q}(\boldsymbol{\zeta}), \boldsymbol{\Psi}_{\phi}(\boldsymbol{\zeta})\right\rangle}{\left\langle\boldsymbol{\Psi}_{\phi}^{2}(\boldsymbol{\zeta})\right\rangle}$.

They are given numerically from a quadrature rule:

$$
\boldsymbol{\lambda}_{q \lambda}=\frac{1}{<\boldsymbol{\Psi}_{\lambda}^{2}(\boldsymbol{\zeta})>} \sum_{i=1}^{n_{\mathrm{gp}}} \boldsymbol{\Lambda}_{q}\left(\boldsymbol{\zeta}_{i}\right) \boldsymbol{\Psi}_{\phi}\left(\boldsymbol{\zeta}_{i}\right) \mathbf{w}_{i}
$$

where:

$$
\boldsymbol{\Lambda}_{q}\left(\boldsymbol{\zeta}_{i}\right)=\sum_{\phi=0}^{n_{\lambda}} \sum_{k=0}^{n_{k}} \sum_{\lambda=0}^{n_{\lambda}} \boldsymbol{\Psi}_{\phi}\left(\boldsymbol{\zeta}_{i}\right) \boldsymbol{\Psi}_{m}\left(\boldsymbol{\zeta}_{i}\right) \boldsymbol{\Psi}_{\lambda}\left(\boldsymbol{\zeta}_{i}\right) \boldsymbol{\phi}_{q \phi}^{\mathrm{T}} \boldsymbol{k}_{k} \boldsymbol{\phi}_{q \lambda} .
$$

An identical strategy enables the computation of modal damping.

\subsection{Determination of the stochastic normal modes basis using the subspace inverse power method and polynomial chaos representation}

Let us now take a look at the stochastic version of the subspace inverse power method presented in the previous subsection 4.1, to see if it is a suitable alternative to the deflated inverse power method in order to build the stochastic normal modes basis.

The proposed algorithm is the following. For an initial set of $n_{r}$ modes $\boldsymbol{\Phi}^{(0)}$ having the PC representation 
$\Phi^{(0)}$, do:

1. Choose $\Phi_{\mathrm{RBM}}$ from null $(\mathbf{k})$ to represent $\boldsymbol{\Phi}_{\mathrm{RBM}}$

2. Normalize $\Phi_{\mathrm{RBM}}$ such that $\boldsymbol{\Phi}_{\mathrm{RBM}}^{\mathrm{T}} \mathbf{M} \boldsymbol{\Phi}_{\mathrm{RBM}}=\mathbf{i}_{0}$

3. From $\phi_{\mathbf{q}}$, define $\Phi_{\mathbf{q}}^{(0)}$ to represents $\boldsymbol{\Phi}_{\mathbf{q}}^{(0)}$ for normal modes corresponding to non null eigenvalues

4. Orthonormalize initial matrix $\Phi^{(0)}$

5. Evaluate $\Lambda_{\mathbf{q}}^{(0)}$ from a modal projection of $\mathbf{K}$ over $\Phi_{\mathbf{q}}^{(0)}$

6. For $k=1,2, \ldots$ do:

(a) Let $\mathcal{F}_{\mathbf{q}}^{(k)}=\mathcal{M} \Phi_{\mathbf{q}}^{(k-1)}$

(b) Solve $\mathcal{K} \Phi_{\mathbf{q}}^{(k)}=\mathcal{F}_{\mathbf{q}}^{(k)}$

(c) Orthonormalize $\boldsymbol{\Phi}^{(k)}$

(d) Evaluate $\Lambda_{\mathbf{q}}^{(k)}$ from a modal projection of $\mathbf{K}$ over $\Phi_{\mathbf{q}}^{(k)}$

7. Let $\Phi_{\mathbf{q}} \leftarrow\left[\Phi_{\mathbf{0}}, \Phi_{\mathbf{q}}\right]$

8. End

In this algorithm, the sub-step (c) implies the orthogonalization of all normal modes, including the rigid body modes, when they exist. As for the stochastic deflated inverse power method, the starting guess $\Phi^{(0)}$ is produced from the normal mode matrix $\phi$ of the deterministic nominal mechanical system obtained by the deterministic subspace inverse power method, and orthonormalization is achieved as in the above subsections 4.4 and 4.3:

1. Evaluate $\Phi_{1}^{(k) 1}$ that normalizes modal mass to unity and let $\Phi_{1}^{(k)} \leftarrow \Phi_{1}^{(k) 1}$

2. For $r \leftarrow 2$ to $n_{r}$,

(a) Find $\Phi_{r}^{(k) \perp}$ that orthogonalizes $\Phi_{r}^{(k)}$ to $\boldsymbol{\Phi}_{p}^{(k)}$ for $p \in\{1, \ldots, r-1\}$ and let $\Phi_{r}^{(k)} \leftarrow \Phi_{r}^{(k) \perp}$

(b) Find $\Phi_{r}^{(k) 1}$ that normalizes the modal mass to unity and let $\Phi_{r}^{(k)} \leftarrow \Phi_{r}^{(k) 1}$

3. End 
and $\max _{r \in\left\{1, \ldots, n_{r}\right\}}\left(\frac{\left\|\Lambda_{r}^{(k)}-\Lambda_{r}^{(k-1)}\right\|}{\left\|\Lambda_{r}^{(k)}\right\|}\right)<\epsilon$.

convergence

condition

for

$\Lambda_{\mathrm{q}}$

reads:

Comparisons of this algorithm with the one proposed for the stochastic version of the deflated inverse power method show that differences occur only in the organization of the numerical operations and resulting computation time but not for obtained results.

\section{$5 \quad$ Numerical applications}

\subsection{First application}

The first application concerns a system having two degree of freedom and involving three random variables. Introducing $\zeta$ for $l=\{1,2,3\}$, three uncorrelated standard normal variables, the random structural matrices are:

$$
\mathbf{K}=\mu_{a}\left(1+\frac{\zeta_{1}^{2}-1}{\sqrt{2}} \frac{\sigma_{a}}{\mu_{a}}\right)\left[\begin{array}{cc}
1 & -1 \\
-1 & 1
\end{array}\right]
$$

and:

$$
\mathbf{M}=\left[\begin{array}{cc}
1 & 0 \\
0 & 1
\end{array}\right]+\frac{1}{\sqrt{2}} \frac{\sigma_{a}}{\mu_{a}}\left[\begin{array}{cc}
\zeta_{2}^{2}-1 & 0 \\
0 & \zeta_{3}^{2}-1
\end{array}\right] .
$$

The parameters are: $\mu_{a}=20$ and $\frac{\sigma_{a}}{\mu_{a}}=5 \%$. Notice that positive definiteness is ensured for the stiffness and masses as long as $\frac{\sigma_{a}}{\mu_{a}}<\sqrt{2}$.

Hermite polynomials up to the second degree (10 terms) are used for the PC representation of the stochastic eigensolutions. Eigensolutions of the nominal system are:

$$
\boldsymbol{\phi}=\frac{1}{\sqrt{2}}\left[\begin{array}{cc}
1 & 1 \\
1 & -1
\end{array}\right] \quad \text { and } \quad \boldsymbol{\lambda}=\left[\begin{array}{cc}
0 & 0 \\
0 & 40
\end{array}\right]
$$

showing that the first normal mode is a rigid body mode. Then, considering the stochastic eigenproblem, the first eigenvalue is deterministic, indeed it is null. The amplitude of the normalized rigid-body mode is however stochastic since it has a unit norm with respect to an inner product defined by the stochastic mass metric. The PC representation of this first normal mode is obtained by normalizing the starting guest which is built from the nominal system. The second normal mode is sought from the deflated inverse power method presented above, using the enhanced strategy for the orthogonal step. Since the iteration matrix $\mathcal{K}$ is not invertible, a Moore-Penrose pseudo inverse is involved for the generation of a new iterate. When starting 


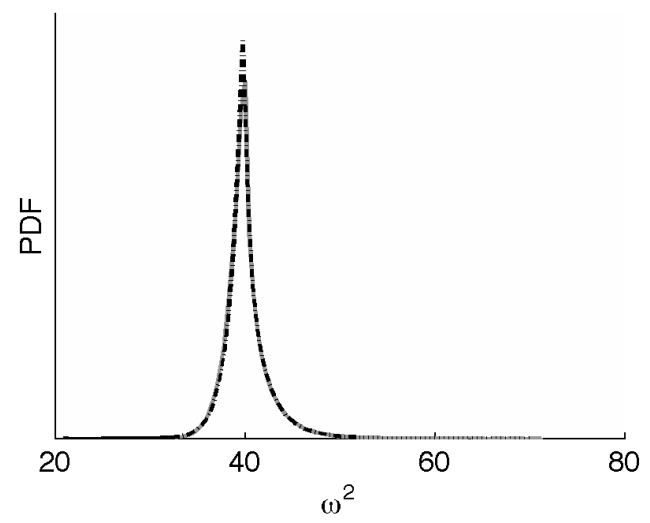

Figure 1: Superimposition of the PDF of the second eigenvalue for the first application; thick grey lines are for MCS, dashed black lines are for PC representation

from the nominal second mode the convergence towards the stochastic second normal mode is reached in two iterations. Figure 1 shows the PDFs of the second eigenvalue and Figure 2 shows the PDFs of the eigenvectors components obtained. These results are compared to a MCS which has been carried out using $5 \times 10^{5}$ sample size. A satisfactory agreement is achieved.

\subsection{Second application}

The second application is inspired by the simplified three DOFs model of the bladed disk of the reference [16]. Introducing $\zeta$ for $l=\{1,2,3,4\}$, four uncorrelated standard normal variables, the random structural matrices are:

$$
\begin{aligned}
\mathbf{K}= & \mu_{b}\left[\begin{array}{lll}
1 & 0 & 0 \\
0 & 1 & 0 \\
0 & 0 & 1
\end{array}\right]+\frac{\sigma_{b}}{\sqrt{2}}\left[\begin{array}{ccc}
\zeta_{1}^{2}-1 & 0 & 0 \\
0 & \zeta_{2}^{2}-1 & 0 \\
0 & 0 & \zeta_{3}^{2}-1
\end{array}\right] \\
& +\mu_{c}\left(1+\frac{\zeta_{4}^{2}-1}{\sqrt{2}} \frac{\sigma_{c}}{\mu_{c}}\right)\left[\begin{array}{ccc}
2 & -1 & 0 \\
-1 & 2 & -1 \\
0 & -1 & 2
\end{array}\right]
\end{aligned}
$$

and:

$$
\mathbf{M}=\left[\begin{array}{ccc}
1 & 0 & 0 \\
0 & 1 & 0 \\
0 & 0 & 1
\end{array}\right]+\frac{1}{\sqrt{2}} \frac{\sigma_{b}}{\mu_{b}}\left[\begin{array}{ccc}
\zeta_{1}^{2}-1 & 0 & 0 \\
0 & \zeta_{2}^{2}-1 & 0 \\
0 & 0 & \zeta_{3}^{2}-1
\end{array}\right]
$$



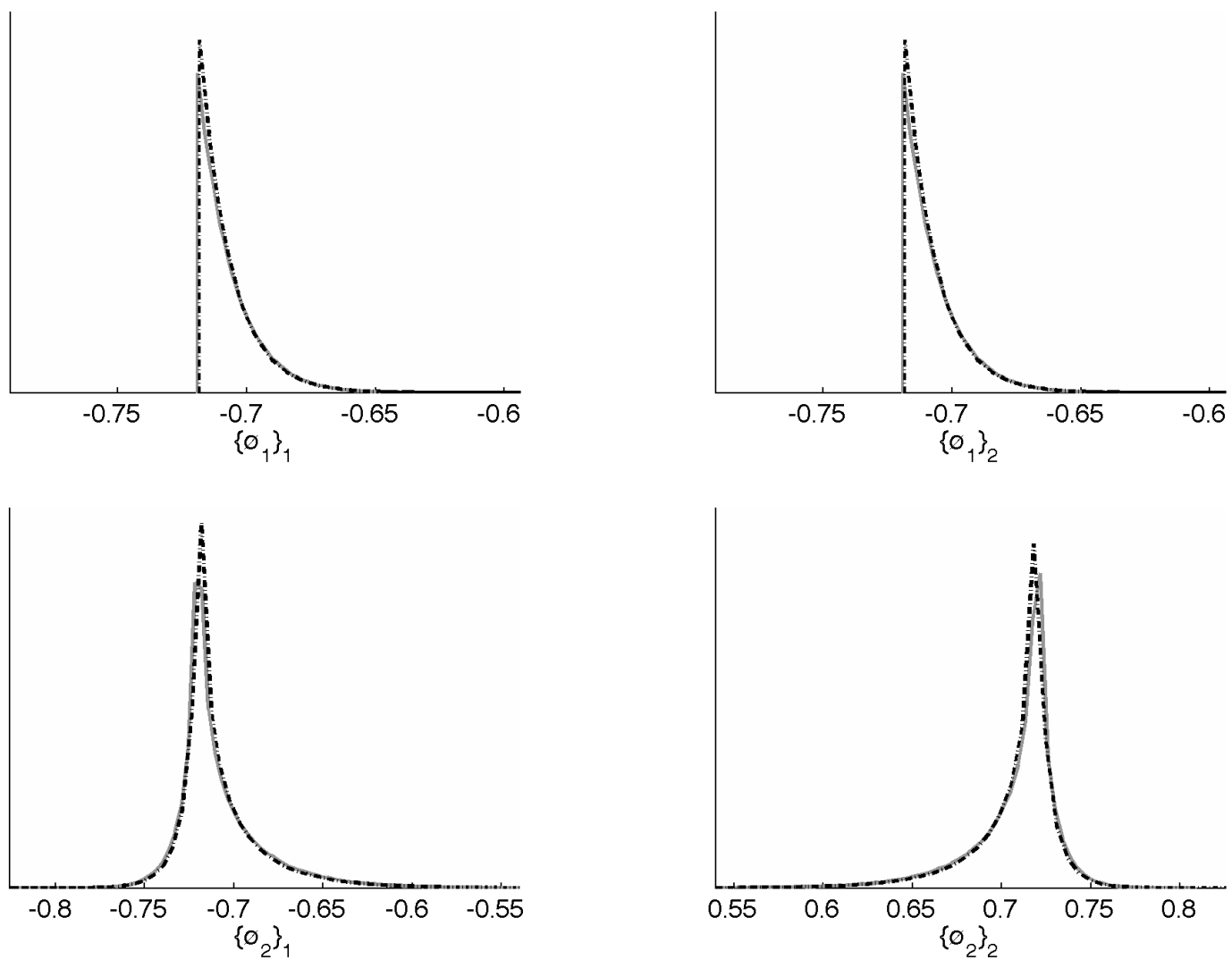

Figure 2: Superimposition of PDFs of the two components (column-wise) of the two normal modes (row-wise) for the first application; thick grey lines are for MCS, dashed black lines are for PC representation 
The parameters used are the ones proposed in the reference [16]: $\mu_{b}=100, \mu_{c}=20$, while $\frac{\sigma_{b}}{\mu_{b}}=\frac{\sigma_{c}}{\mu_{c}}=5 \%$ and Hermite polynomials are chosen for the PC representation of the stochastic eigensolutions and for the PC representation of the stochastic dynamic responses. The deflated inverse power method presented above is chosen to find the three stochastic normal modes while the normal modes of the nominal system are used as starting guesses. These normal modes are:

$$
\boldsymbol{\phi}=\frac{1}{2}\left[\begin{array}{ccc}
1 & \sqrt{2} & 1 \\
\sqrt{2} & 0 & -\sqrt{2} \\
1 & -\sqrt{2} & 1
\end{array}\right]
$$

Reference results are produced by MCS carried out using $5 \times 10^{5}$ sample size and a Modal Assurance Criterion to identify and to order the stochastic normal modes. Empirical means and standard deviations of stochastic eigenvalues are given in Table 1 and their PDFs are plotted in Figure 3. Empirical CDFs of $\left\{\Phi_{r}\right\}_{i}$, the components $i$ of the normal modes $\Phi_{r}$, are shown in Figure 4 . These MCS results are reference results to be compare with the one produced by the PC representation. For the current application, the 3rd component of the 3rd normal mode is the most critical regarding the convergence issue. For this normal mode component, if the 2 nd degree ( 15 terms) or the 3rd degree (35 terms) is chosen for the polynomial order involved in PC representation, we get an identical relative error of $50 \%$ in the skewness and a relative error of $75 \%$ in the kurtosis of this normal mode component. But for a fourth degree (70 terms), we get a very satisfactory agreement between MCS and PC representation, as shown in Figure 4. In this case, the deflated inverse power algorithm presented above requires 10,14, and 2 iterations to converge to a relative precision $\epsilon=10^{-6}$ for each stochastic eigenfrequency of the normal modes.

To compute frequency responses functions, the structural forces and damping matrices are $\mathbf{f}(\omega)=$ $\left[\begin{array}{lll}1 & 0 & 0 \\ 0 & 1 & 0 \\ 0 & 0 & 1\end{array}\right]$ and $\mathbf{C}=\left[\begin{array}{ccc}1 & 0 & 0 \\ 0 & 1 & 0 \\ 0 & 0 & 1\end{array}\right]$. Figure 5 and 6 show i) the mean; ii) the 5 percentile; and iii) the 95 percentile of the amplitude of the nine frequency responses functions, as well as their second statistical moment. Notice that computing the mean amplitude requires all the terms of the PC representation, and not only the first one. Hence, agreement with MCS can only be achieved for an adequate truncation of the PC representation. For the stochastic eigenproblem, a satisfactory agreement is achieved. However, small discrepancies can be observed at $\omega^{2} \simeq 110$ and $\omega^{2} \in[165,175]$. This indicates that the order of truncation is not totally adequate in these ranges for the frequency responses, while it is adequate for the stochastic 
Table 1: Statistics of normal modes frequencies for the second application

\begin{tabular}{|c|c|c|c|}
\hline Normal mode number $r$ & 1 & 2 & 3 \\
\hline \hline Mean of frequency & 111.7 & 140.0 & 168.5 \\
\hline Standard deviation of frequency & 0.681 & 2.40 & 3.87 \\
\hline
\end{tabular}

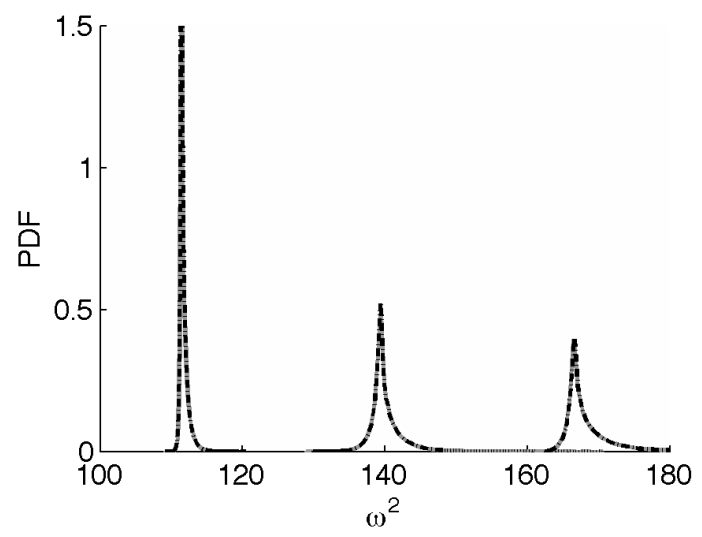

Figure 3: Superimposition of PDFs of the three eigenvalues for the second application; thick grey lines are for MCS, dashed black lines are for PC representation

normal modes.

\subsection{Third application}

The third application is the simplified three DOFs model of the bladed disk of the reference [16]. This application is interesting as two of the three deterministic normal modes have the same natural frequency. Introducing $\zeta$ for $l=\{1,2,3,4\}$, four uncorrelated standard normal variables, the random structural matrices are:

$$
\begin{aligned}
& \mathbf{K}=\mu_{b}\left[\begin{array}{ccc}
1 & 0 & 0 \\
0 & 1 & 0 \\
0 & 0 & 1
\end{array}\right]+\frac{\sigma_{b}}{\sqrt{2}}\left[\begin{array}{ccc}
\zeta_{1}^{2}-1 & 0 & 0 \\
0 & \zeta_{2}^{2}-1 & 0 \\
0 & 0 & \zeta_{3}^{2}-1
\end{array}\right] \\
& +\mu_{c}\left(1+\frac{\zeta_{4}^{2}-1}{\sqrt{2}} \frac{\sigma_{c}}{\mu_{c}}\right)\left[\begin{array}{ccc}
2 & -1 & -1 \\
-1 & 2 & -1 \\
-1 & -1 & 2
\end{array}\right]
\end{aligned}
$$

and $\mathbf{M}$ is the identity. The parameters are: $\mu_{b}=100, \mu_{c}=20$, while $\frac{\sigma_{b}}{\mu_{b}}=\frac{\sigma_{c}}{\mu_{c}}=5 \%$ and Hermite polynomials up to the fourth degree are used for the PC representation of the stochastic eigensolutions and for the PC 

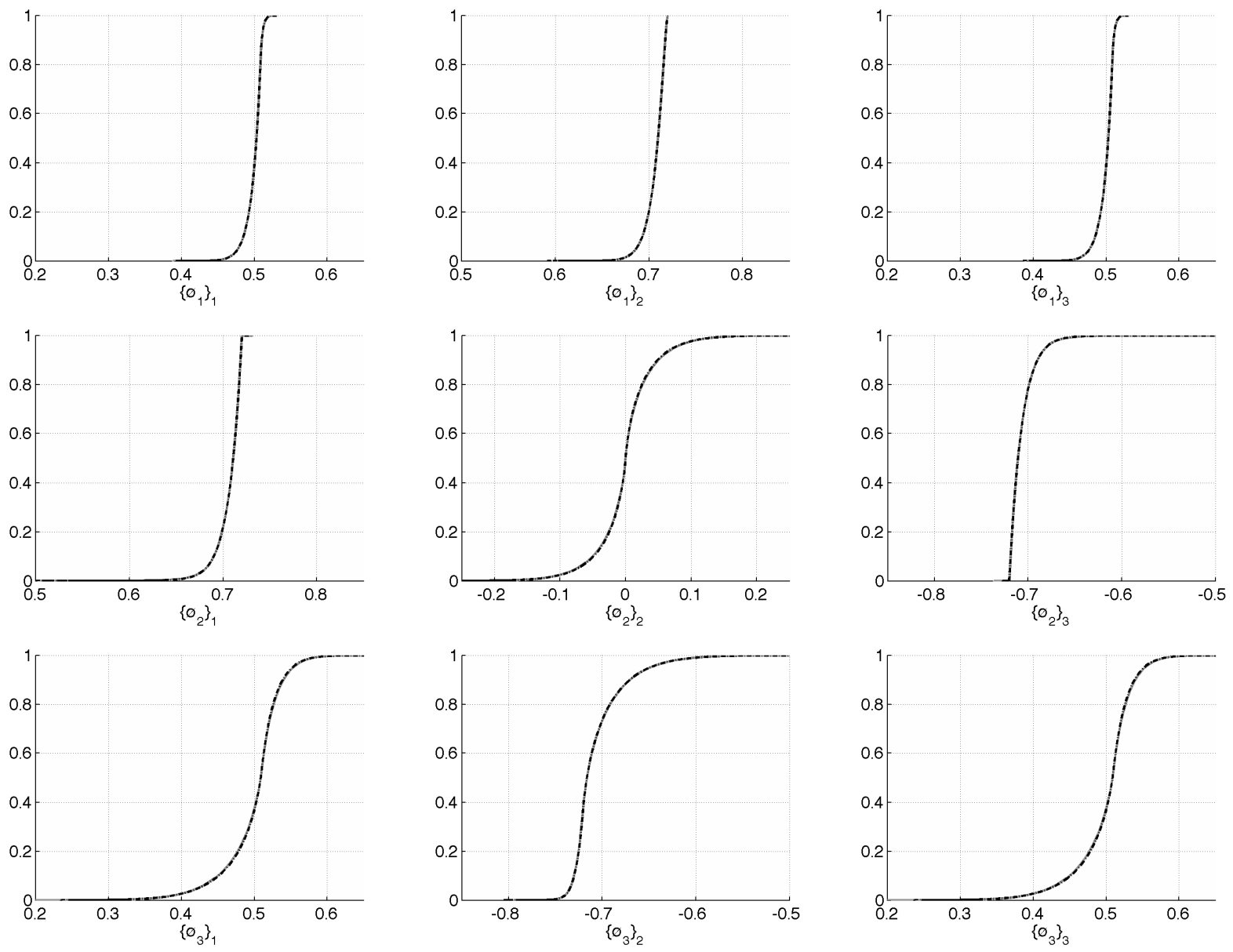

Figure 4: Superimposition of CDFs of the three components (column-wise) of the three normal modes (rowwise) for the second application; thick grey lines are for MCS, dashed black lines are for PC representation 

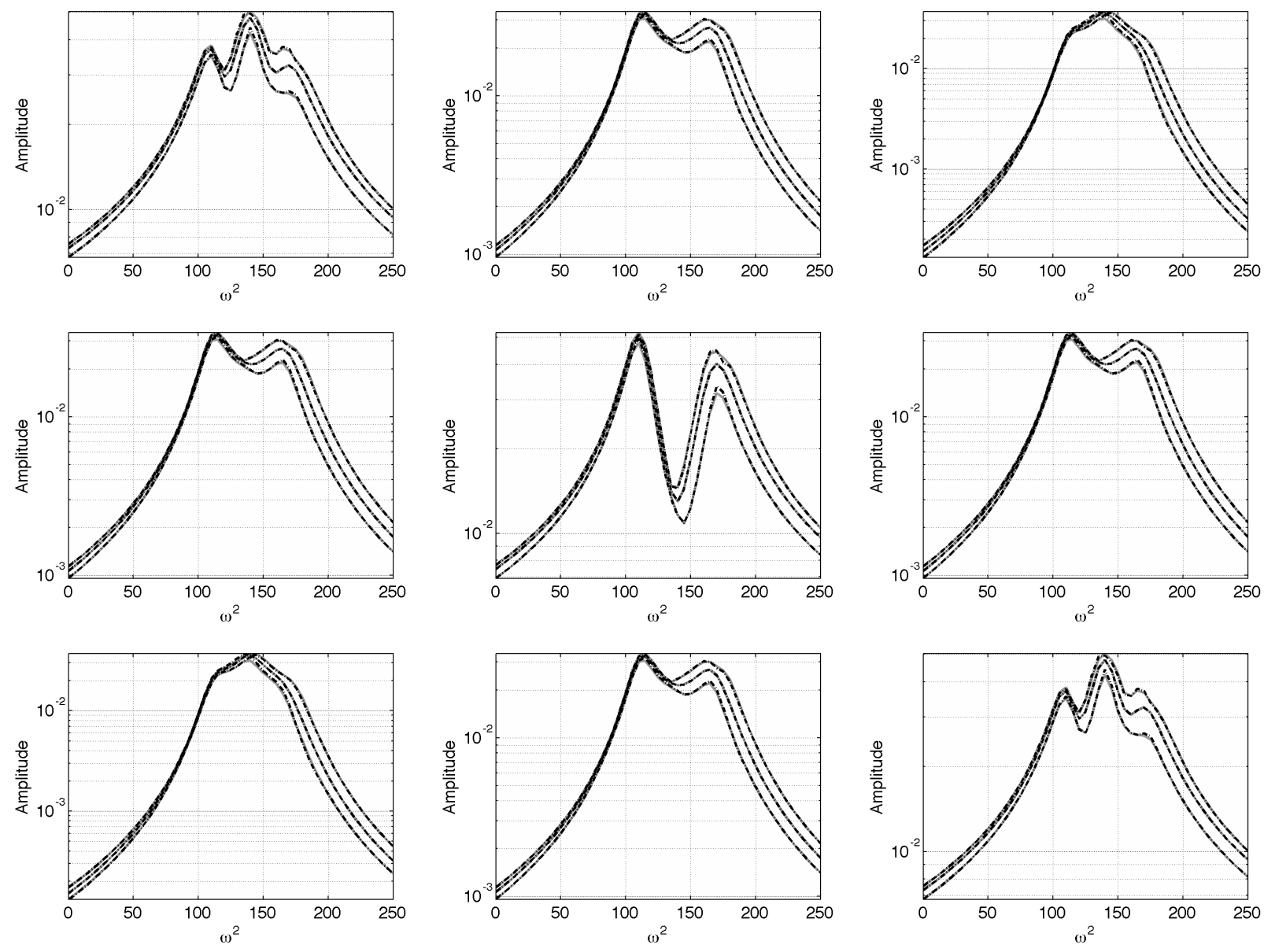

Figure 5: Superimposition of frequency responses amplitudes of the three DoF system for the second application using the PC representation up to the 4th degree; Mean, $5 \%$ and $95 \%$ lines of confidence interval are represented; From up to down is for the first to the last degree of freedom; From left-hand to right-hand is for an excitation applied from the first to the last degree of freedom; Thick grey lines are for MCS, dashed black lines are for PC representation 

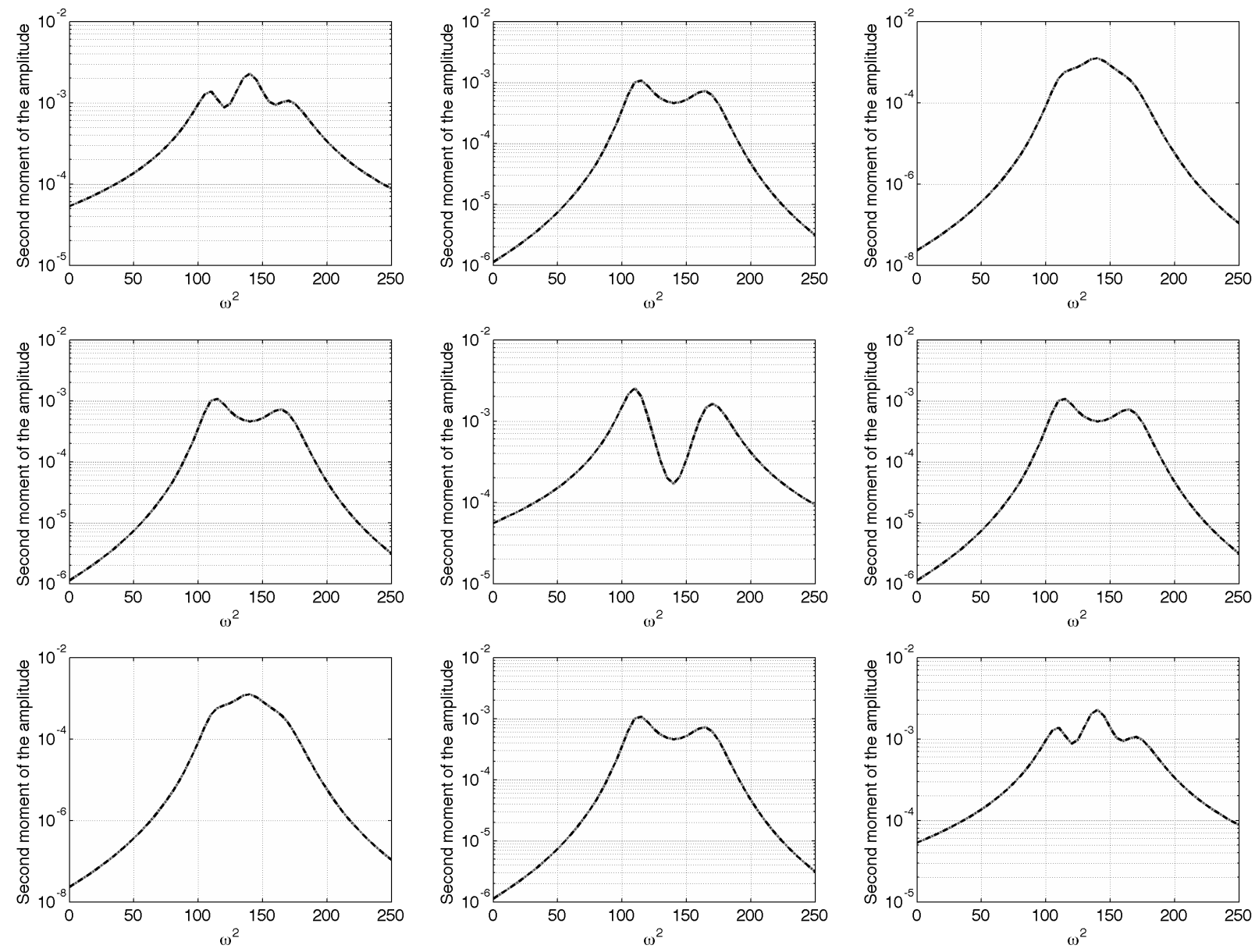

Figure 6: Superimposition of second moment of frequency responses amplitudes of the three DoF system for the second application using the $\mathrm{PC}$ representation up to the 4th degree; From up to down is for the first to the last degree of freedom; From left-hand to right-hand is for an excitation applied from the first to the last degree of freedom; Thick grey lines are for MCS, dashed black lines are for PC representation 
Table 2: Statistics of normal modes frequencies for the third application

\begin{tabular}{|c|c|c|c|}
\hline Normal mode number $r$ & 1 & 2 & 3 \\
\hline \hline Mean of frequency & 99.75 & 159.4 & 160.8 \\
\hline Standard deviation of frequency & 2.51 & 4.55 & 5.54 \\
\hline
\end{tabular}

representation of the stochastic dynamic responses. Eigensolutions of the nominal system are:

$$
\boldsymbol{\phi}=\frac{1}{6}\left[\begin{array}{ccc}
2 \sqrt{3} & \sqrt{6} & 3 \sqrt{2} \\
2 \sqrt{3} & \sqrt{6} & -3 \sqrt{2} \\
2 \sqrt{3} & -2 \sqrt{6} & 0
\end{array}\right] \quad \boldsymbol{\lambda}=\left[\begin{array}{ccc}
100 & 0 & 0 \\
0 & 160 & 0 \\
0 & 0 & 160
\end{array}\right]
$$

showing that the second and the third natural frequencies are equals. When starting from these eigenvectors of the nominal system, the stochastic deflated inverse power method presented above find the three stochastic modes using 10, 82 and 11 iterations for a relative precision $\epsilon=10^{-6}$ for each eigenfrequency. Results of means and standard deviations of stochastic eigenvalues obtained are given in Table 2.

To compute frequency responses functions, the structural forces, and the damping matrices are $\mathbf{f}(\omega)=$ $\left[\begin{array}{lll}1 & 0 & 0 \\ 0 & 1 & 0 \\ 0 & 0 & 1\end{array}\right]$ and $\mathbf{C}=\left[\begin{array}{ccc}1 & 0 & 0 \\ 0 & 1 & 0 \\ 0 & 0 & 1\end{array}\right]$

Figures 7 and 8 show i) the mean; ii) the 5 percentile; and iii) the 95 percentile of the amplitudes of the nine frequency response functions, as well as their second statistical moments. The obtained results are compared to a MCS which has been carried out using $5 \times 10^{5}$ sample size. A satisfactory agreement is achieved, especially around the resonant frequencies, showing that eigenvalues multiplicity is handled effectively.

\subsection{Fourth application}

The fourth application is a realistic mechanical situation by considering a problem where more degrees of freedom than the number of sough normal modes while repeated eigenvalues exist for the nominal system. Its purpose is to show the applicability of the strategies presented above to obtain modal based frequency responses in the low frequency range.

This application addresses frequency response functions of a simplified tower guyed structure. More precisely the responses in the plane $\mathcal{O} x y$ at the guys connection point $\mathcal{P}$ are of interest, see Fig. 9. Overall dimensions are such that eigenvalues are repeated and close to each other. For a guyed mast, wind and ice are the major loads which alter the nominal tension in guys. Thus, in this application, tensions in guys are 

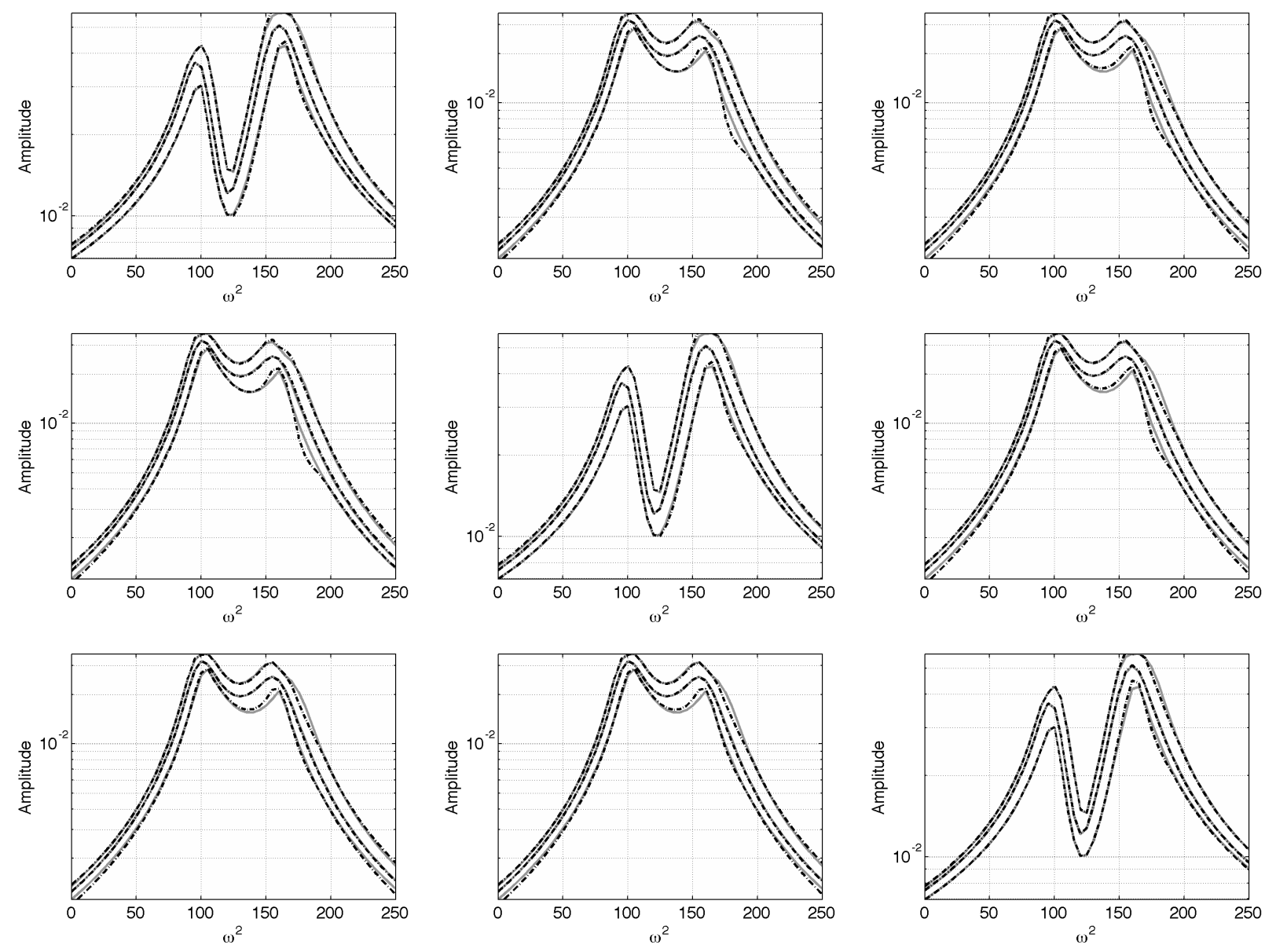

Figure 7: Superimposition of frequency responses amplitudes of the three DoF system for the third application using the PC representation up to the 4th degree; Mean, 5\% and $95 \%$ lines of confidence interval are represented; From up to down is for the first to the last degree of freedom; From left-hand to right-hand is for an excitation applied from the first to the last degree of freedom; Thick grey lines are for MCS, dashed black lines are for PC representation 

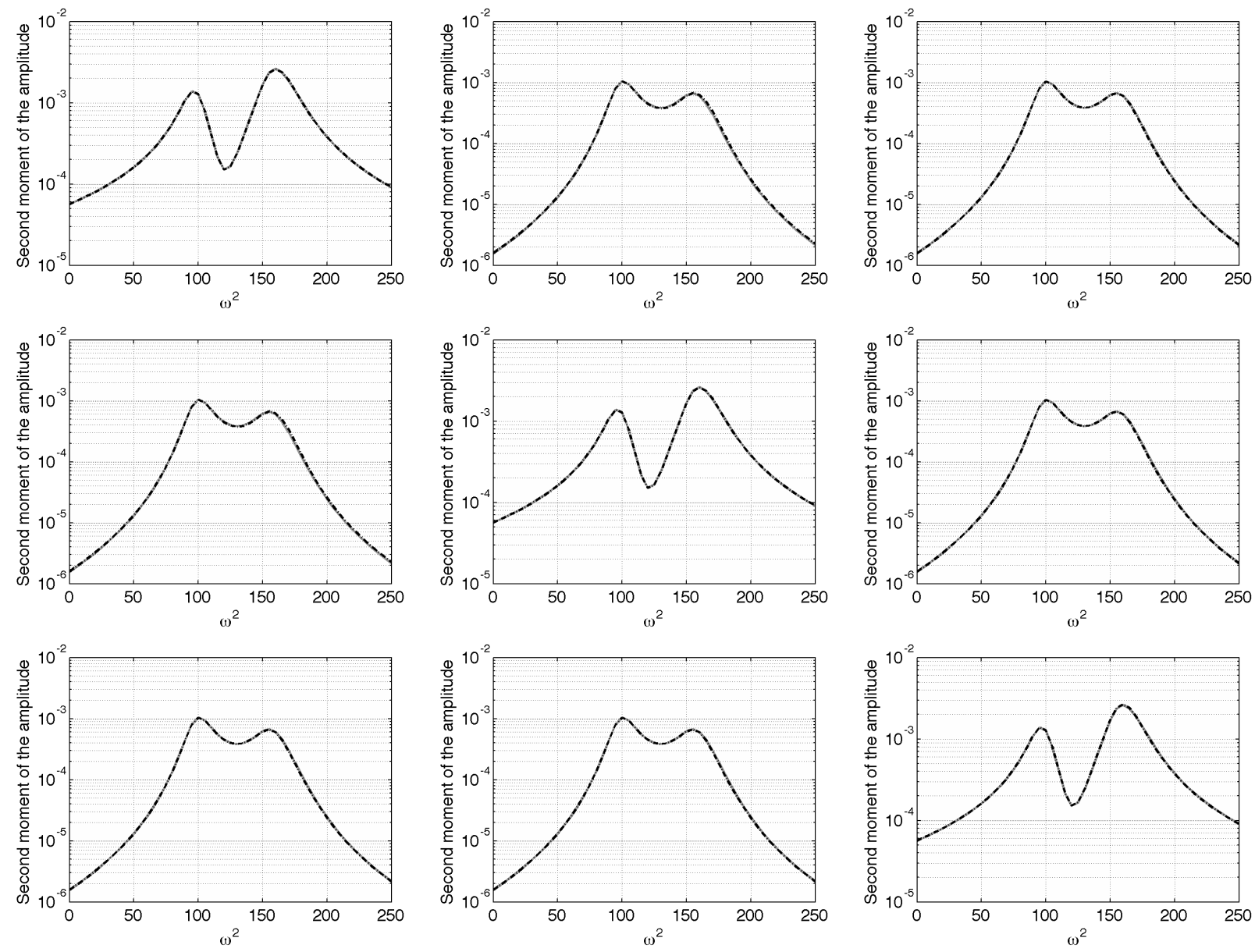

Figure 8: Superimposition of second moment of frequency responses amplitudes of the three DoF system for the third application using the PC representation up to the 4th degree; From up to down is for the first to the last degree of freedom; From left-hand to right-hand is for an excitation applied from the first to the last degree of freedom; Thick grey lines are for MCS, dashed black lines are for PC representation 
supposed stochastic and we are interested with uncertainties quantification in frequency response functions of the $\mathcal{P}$ point.

The proposed simplified guyed mast structure consists of a vertical mast-column and 3 guys (Fig. 9). The mast is pinned at its base and guyed in three directions at $120^{\circ}$ angles in the plan. Guys are connected to the mast at the $\mathcal{P}$ point located at a height of $h=135 \mathrm{~m}$. Distance between guy anchors and base tower is $d=145 \mathrm{~m}$. Guys nominal tensions are $t=40,000 \mathrm{~N}$ at guy anchors. Their Young's modulus is $e=165$ GPa, mass density is $\rho=8,100 \mathrm{~kg} / \mathrm{m}^{3}$ and area $a=3 \times 10^{-4} \mathrm{~m}^{2}$.

Guy is a tensioned cable and cable structures are nonlinear elastic systems with large displacements. If we consider the cable in the $\mathcal{O} x z$ plane, the static equilibrium of the cable subject to tension and self weight leads to the profile of the cable which may be described by [23]:

$$
z=\frac{t_{x}}{q} \cosh \left(\frac{q}{t_{x}} x+a_{1}\right)-\frac{t_{x}}{q} \cosh \left(a_{1}\right)+h
$$

where $t_{x}$ is the horizontal component of the cable tension, $q=-9.81 \rho a$ is the distributed self weight of the cable and:

$$
a_{1}=\operatorname{arcsinh}\left(\frac{q h}{2 t_{x} \sinh \left(\frac{q d}{2 t_{x}}\right)}\right)-\frac{q d}{2 t_{x}} .
$$

In addition, the tension at any point on the cable is given by:

$$
t_{s}=t_{x} \cosh \left(\frac{q}{t_{x}} x+a_{1}\right)
$$

hence $t=t_{x} \cosh \left(\frac{q}{t_{x}} d+a_{1}\right)$.

Once the static equilibrium of guys is achieved, the geometry of the system is well defined and additional displacements and strains due to dynamic loads are supposed to be small. Thus, a dynamic linear behavior is assumed. Guys are then modeled by a serie of 20 truss finite elements producing a mass matrix and a stiffness matrix composed by the sum of an elastic stiffness matrix and a geometric stiffness matrix [21]. For the response in the $\mathcal{O} x y$ plane, the mast contribution to the stiffness is neglected. However, partial mass of the mast act on node $\mathcal{P}$. It is modeled as a single lumped mass, being $5,750 \mathrm{~kg}$. The resulting global assembly leads to a system having the form of Eq. (1) where the damping matrix is $\mathbf{c}=0.05 \mathbf{m}$ and the number of active degrees of freedoms for the finite element model is $n=173$. The deterministic modal analysis with nominal tensions exhibit repeated or close eigenvalues: $\lambda_{1}=\lambda_{2}=3.04 \mathrm{rd}^{2} / \mathrm{s}^{2}, \lambda_{3}=3.29 \mathrm{rd} / \mathrm{s}^{2}$, $\lambda_{4}=\lambda_{5}=3.46 \mathrm{rd}^{2} / \mathrm{s}^{2}$, see Fig. 10, associated to damped factors $\frac{1}{2 \sqrt{\lambda_{1}}} \xi_{1}=\frac{1}{2 \sqrt{\lambda_{2}}} \xi_{2}=1.43 \%, \frac{1}{2 \sqrt{\lambda_{3}}} \xi_{3}=1.38$ 
$\%, \frac{1}{2 \sqrt{\lambda_{4}}} \xi_{4}=\frac{1}{2 \sqrt{\lambda_{5}}} \xi_{5}=1.34 \%$. It is chosen to approximate the frequency responses functions by truncating arbitrary the modal basis to the five first normal modes.

For a tower guyed, wind and ice are the major loads. The wind can increase the tension in one guy while it can decrease the tension in another guy. In contrast, the ice will result in an increase for tensions in all guys. Then, the 3 tensions in guys are supposed to be random and correlation between them is a parameter of the study. For the 3 random variables, we choose arbitrary to consider a uniform law having a $4.5 \%$ coefficient of variation (being the standard deviation over the mean) while the mean is fixed at the nominal value $t$. Then, three situations are considered for correlation coefficients of the random variables:

1. $\rho_{i j}=0$ for $i \neq j, i \in\{1,2,3\}$ and $j \in\{1,2,3\}$.

2. $\rho_{i j}=-0.4$ for $i \neq j, i \in\{1,2,3\}$ and $j \in\{1,2,3\}$.

3. $\rho_{i j}=1$ for $i \in\{1,2,3\}$ and $j \in\{1,2,3\}$.

Accordingly with the uniform variables, Legrendre polynomials are chosen for the $\mathrm{PC}$ representation. We chose to truncate the polynomial basis at an order 8, leading to 165 polynomials terms and coefficients and 28, 545 unknowns for the system of Eq. (10).

Figures 12, 13 and 14 show the mean; and ii) the 5 and the 95 percentile of the amplitudes of the two horizontal frequency response functions, as well as their second statistical moments, for the correlation coefficients of cases 1 to 3 (respectively). The obtained results are compared to MCSs which have been carried out using $10^{6}$ sample size. A satisfactory agreement is achieved, even around the resonant frequencies for all these 3 cases. Thus, meaningful results can be effectively achieved by the proposed strategies even for such complex situations which involve close and repeated eigenvalues.

\section{Summary}

This work concerns modal frequency responses of stochastic discretized linear dynamic problems. It is limited to symmetrical structural matrices and proportional damping. The PC representation is the chosen spectral approach for random quantities. Within this framework, from the normal basis construction up to the modal synthesis, we propose efficient formulations and strategies in order to maintain the computational effort similar to the one involved for stochastic linear static problems.

To treat the stochastic eigenproblem, instead of a newton-raphson strategy, adaptation of the deterministic deflated inverse power and subspace inverse power method is achieved in the stochastic framework. The 


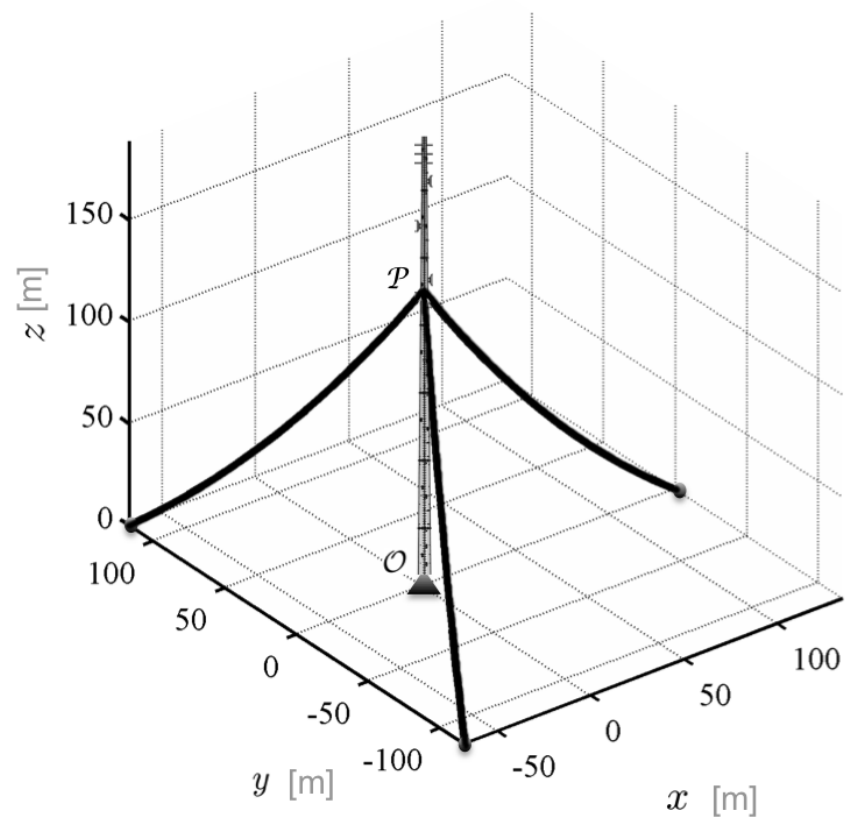

Figure 9: Squetch of the guyed tower

deterministic subspace inverse power method which deal effectively with close and repeated eigenvalues is chosen to initiate starting guess of both these iterative algorithms. Examination of involved steps of both these stochastic versions shows that they both have the advantage of having a main step which involves the resolution of a linear system similar to the one produces by the static case. Thus, as for linear stochastic static problems, required expectations are limited to three PC products for this computation step. Moreover, we have pay special attention to propose efficient numerical formulations or strategies for ensuring orthonormalization of the modal basis and for obtaining modal based frequency responses. They also use only expectations of PC products which are already used for stochastic static problems. All these propositions ensure the numerical efficiency of the stochastic modal based frequency response analysis.

Next, three simple applications and one realistic situation are studied. MCS is avoided to initiate iterations of the stochastic deflated inverse power algorithm. As a starting guesses, eigensolutions of the deterministic nominal problems are chosen. Through these applications, applicability of the proposed strategies to obtain modal based frequency responses is shown even for the realistic situation where there is more degrees of freedom than the number of sough normal modes while repeated eigenvalues exist for the nominal system. It is also shown that the proposed method can handle null eigenvalues. Comparisons of obtained results with the ones computed with MCS are satisfactory. In addition, it is found that frequency responses are more 


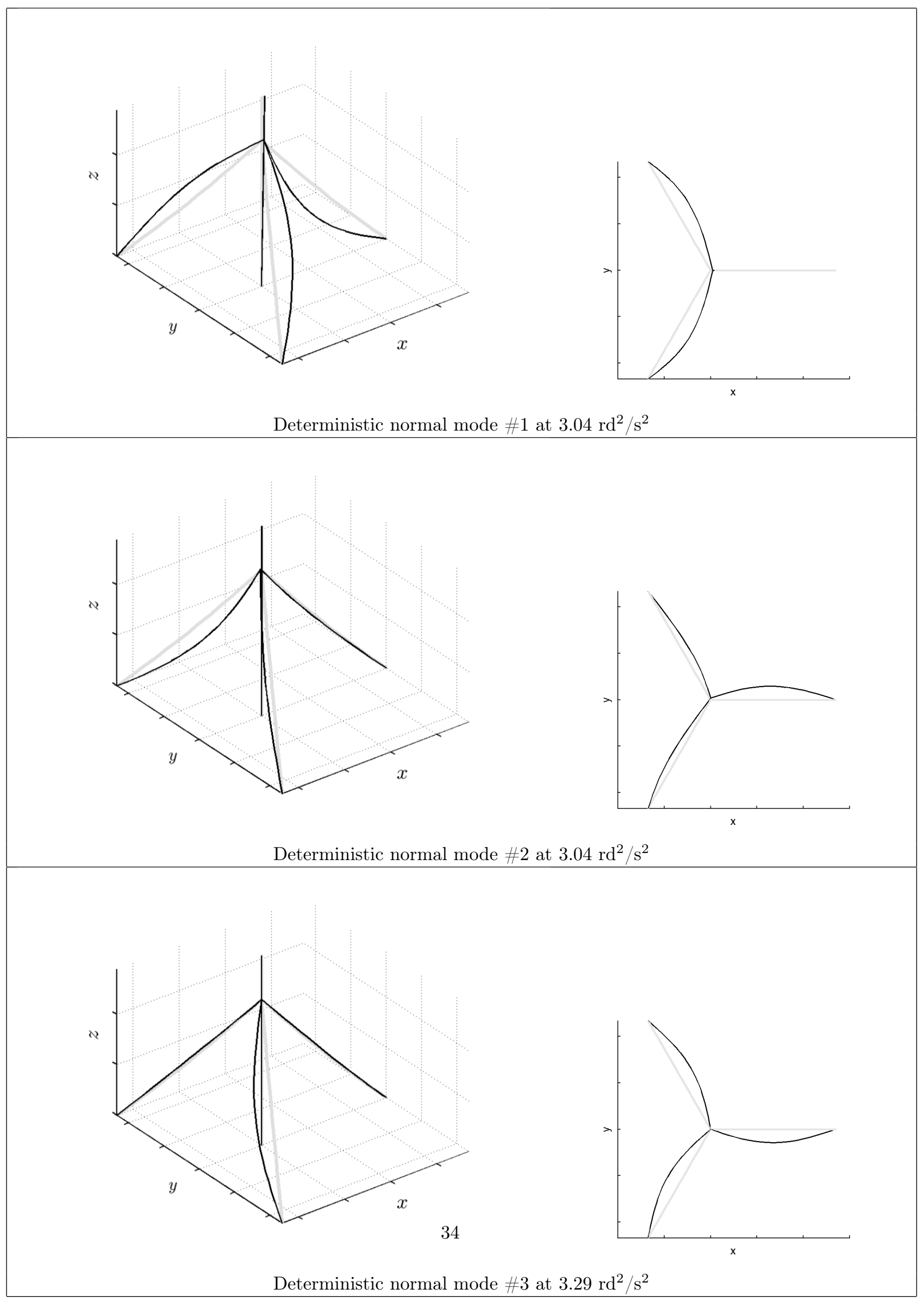

Figure 10: Guyed tower deterministic normal modes of the fourth application; Grey lines are for the unde- 


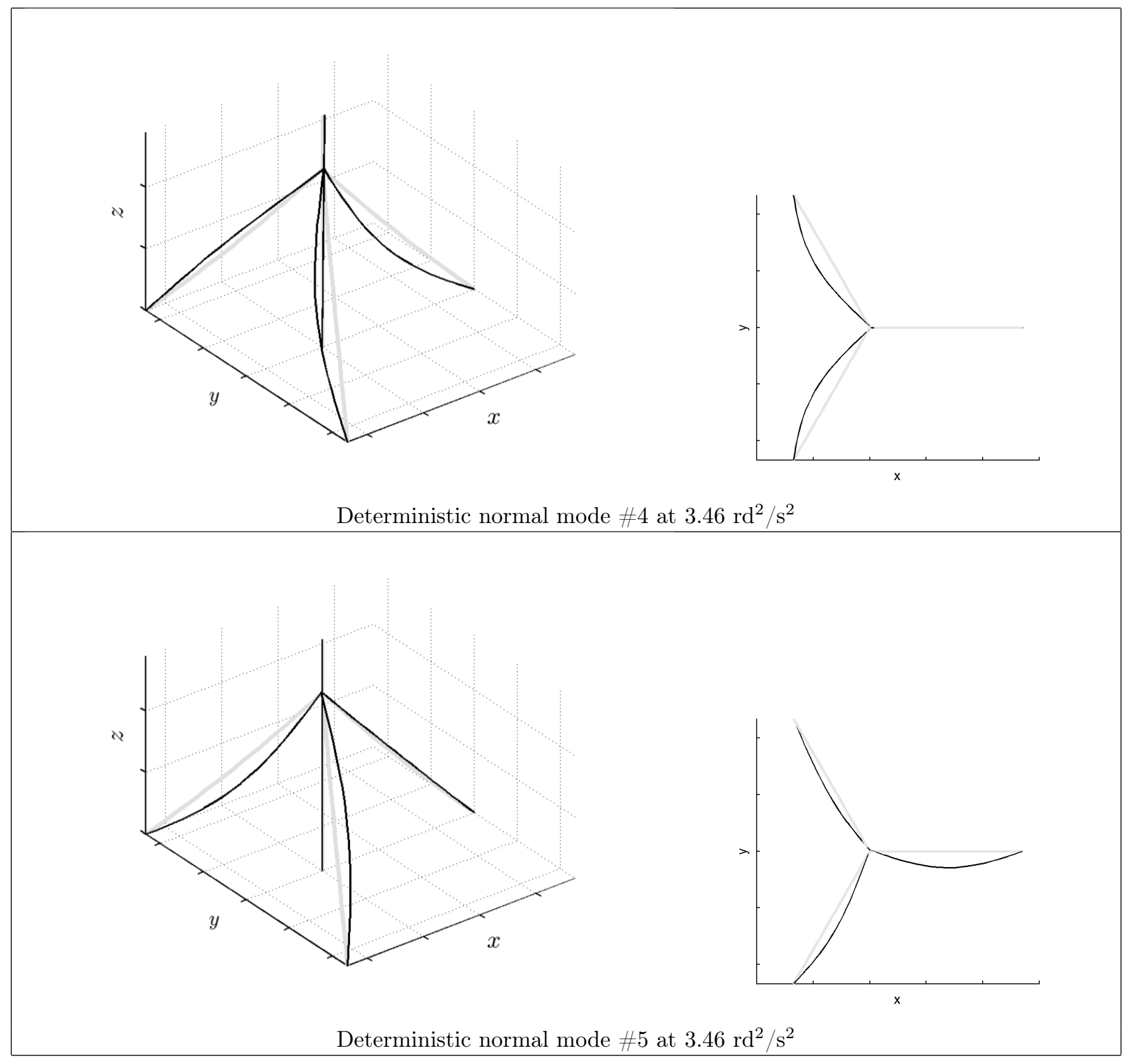

Figure 11: (continued) Guyed tower deterministic normal modes of the fourth application; Grey lines are for the undeformed position, black lines are for the deformed position 

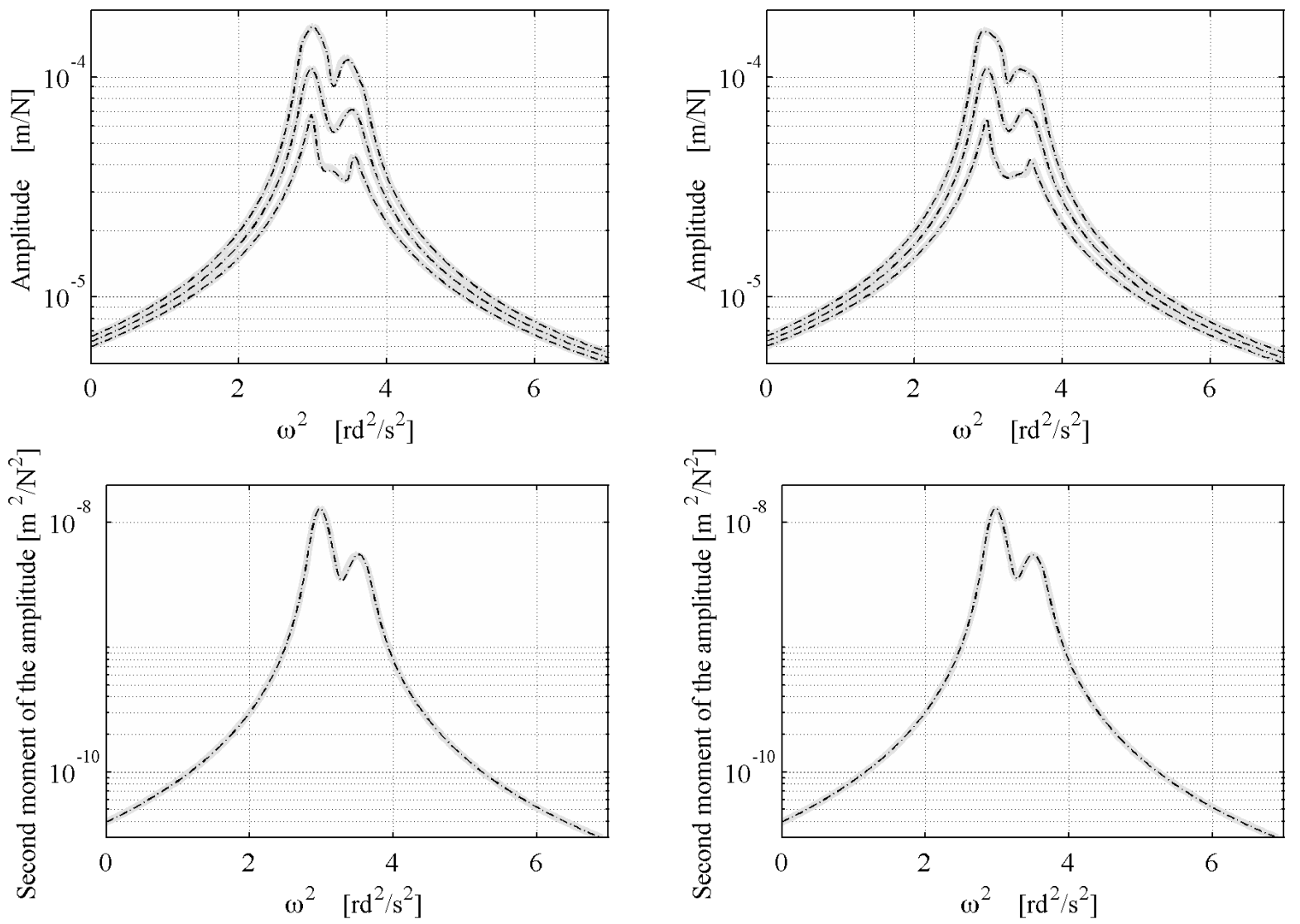

Figure 12: Case 1 results for the guyed tower of the fourth application using the PC representation up to the 8th degree (dash-dot black lines) vs. MCS (continuous thick grey lines); Up: mean of the amplitudes of the 2 frequency responses functions, as well as the 5\% and $95 \%$ lines of confidence interval; Down: second moment of the amplitude of the 2 frequency responses functions; Left subfigures concern the response in the $x$-direction due to an excitation in the $x$-direction; Right subfigures concern the response in the $y$-direction due to an excitation in the $y$-direction 

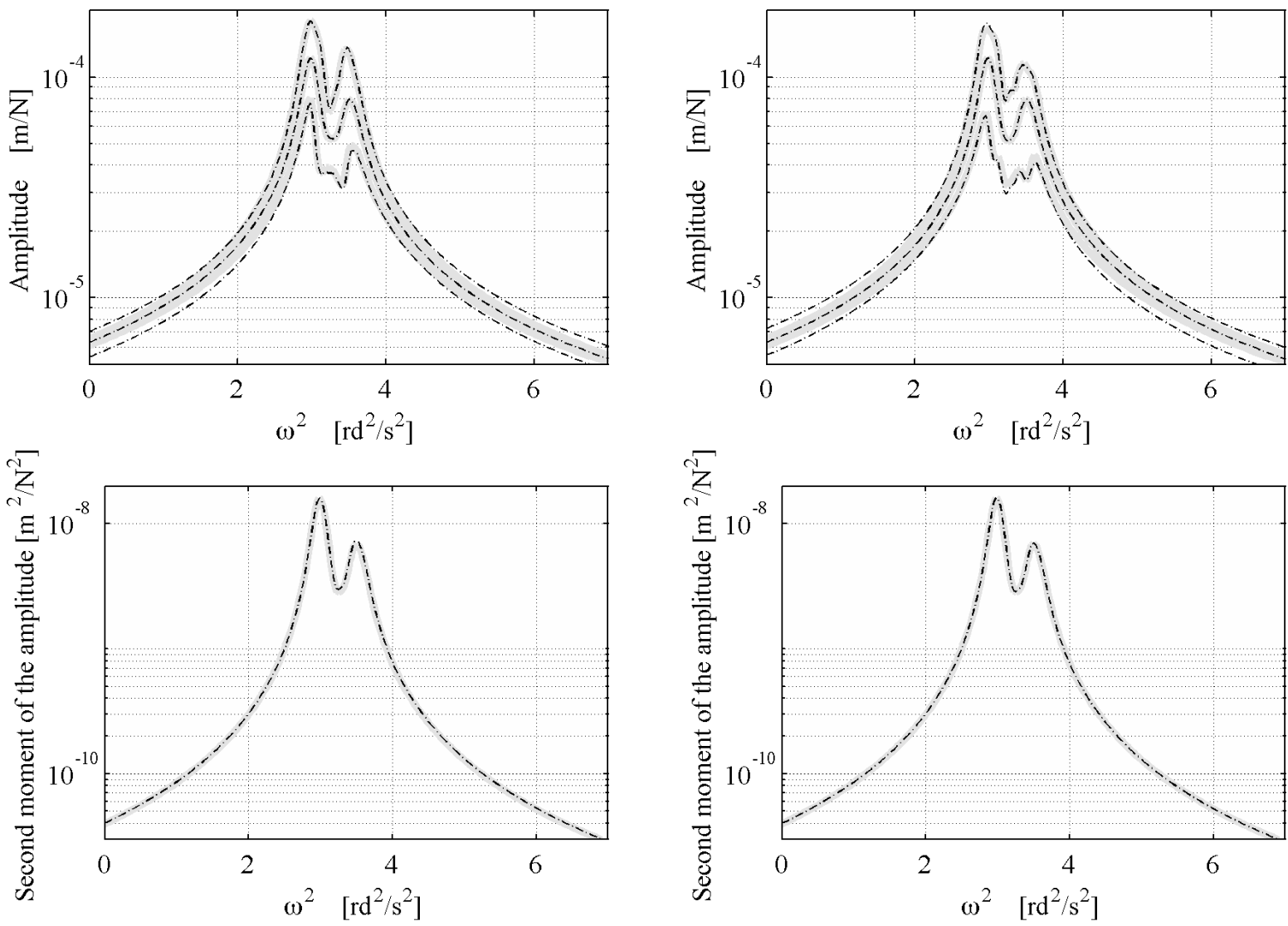

Figure 13: Case 2 results for the guyed tower of the fourth application using the PC representation up to the 8th degree (dash-dot black lines) vs. MCS (continuous thick grey lines); Up: mean of the amplitudes of the 2 frequency responses functions, as well as the 5\% and $95 \%$ lines of confidence interval; Down: second moment of the amplitude of the 2 frequency responses functions; Left subfigures concern the response in the $x$-direction due to an excitation in the $x$-direction; Right subfigures concern the response in the $y$-direction due to an excitation in the $y$-direction 

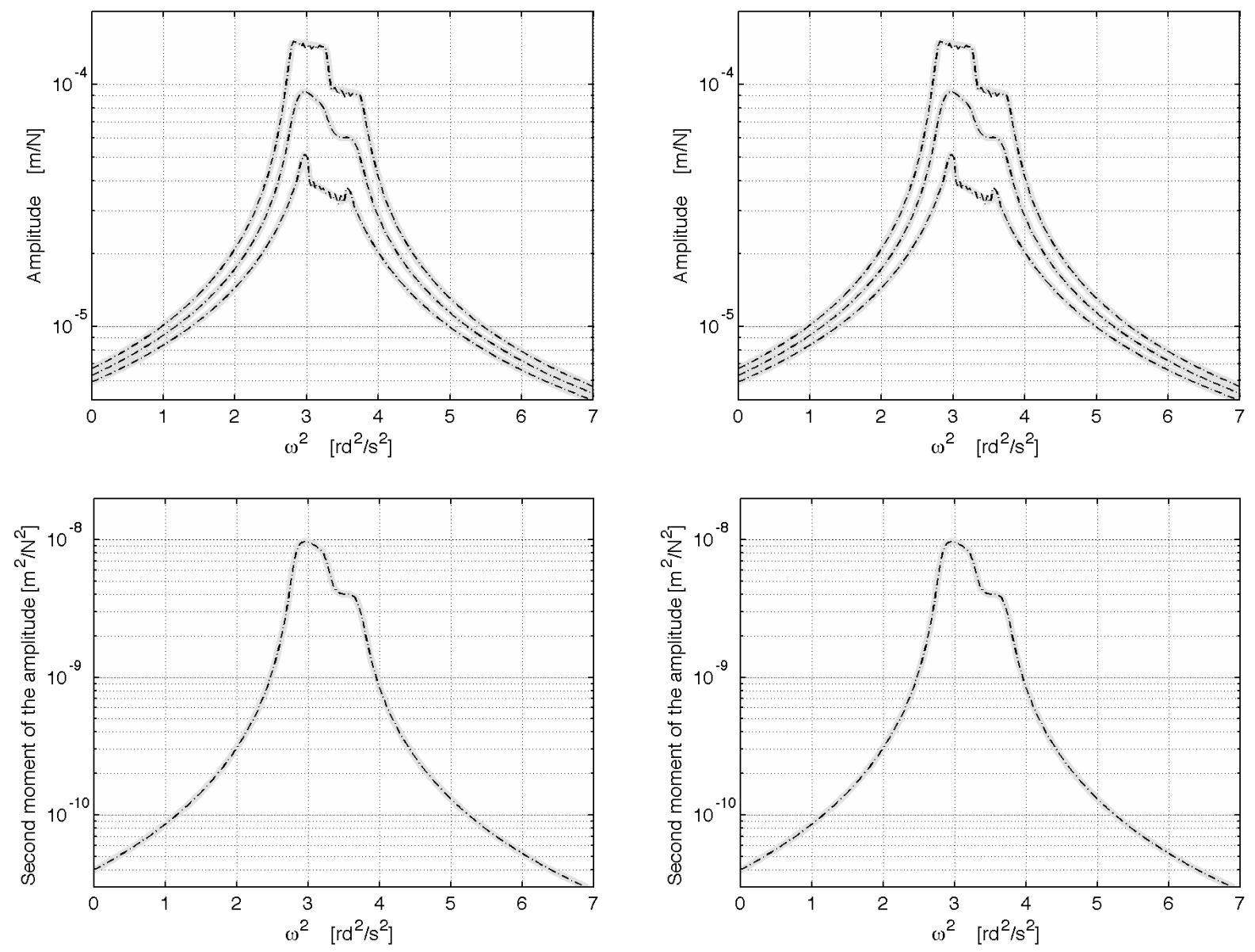

Figure 14: Case 3 results for the guyed tower of the fourth application using the PC representation up to the 8th degree (dash-dot black lines) vs. MCS (continuous thick grey lines); Up: mean of the amplitudes of the 2 frequency responses functions, as well as the $5 \%$ and $95 \%$ lines of confidence interval; Down: second moment of the amplitude of the 2 frequency responses functions; Left subfigures concern the response in the $x$-direction due to an excitation in the $x$-direction; Right subfigures concern the response in the $y$-direction due to an excitation in the $y$-direction 
difficult to represent with $\mathrm{PC}$ than the stochastic normal modes.

\section{References}

[1] Collins JD, Thomson WT. (1969) The eigenvalue problem for structural systems with statistical properties. AIAA Journal 7(4):642-648.

[2] Hart GC, Collins JD. (1970) The treatment of randomness in finite element modelling. SAE Shock and Vibrations Symposium, Los Angeles, CA, 2509-2519.

[3] Shinozuka M, Astill CJ. (1972) Random eigenvalue problems in structural analysis. AIAA Journal 10(4):456-462.

[4] Dessombz O, Diniz A, Thouverez F, Jézéquel L. (1999) Analysis of stochastic structures: perturbation method and projection on homogeneous chaos. Proceedings of the IMAC XVII, Kissimmee, FL.

[5] Szekely GS, Schueller GI. (2001) Computational procedure for a fast calculation of eigenvectors and eigenvalues of structures with random properties. Computer Methods in Applied Mechanics and Engineering 191(8): 799-816.

[6] G.I. Schuëller, H.J. Pradlwarter (2009) Uncertain linear systems in dynamics: Retrospective and recent developments by stochastic approaches, Engineering Structures, 31(11), 2507 - 2517

[7] Nair P.B., Keane A. (2000) New developments in computational stochastic mechanics, part ii applications. In 41st AIAA/ASME/ASCE/AHS/ASC Structures, Structural Dynamics and Materials Conference. AIAA, Atlanta, GA. AIAA 2000-1441.

[8] Nair P.B., Keane A. (2002) Stochastic reduced basis methods. AIAA Journal, 40(8):1653-1664

[9] Ghosh D, Ghanem RG (2005) A new algorithm for solving the random eigenvalue problem using polynomial chaos expansion. 46th AIAA/ASME/ASCE/AHS/ASC Structures, Structural Dynamics and Material Conference, Austin, Texas

[10] Ghanem RG, Ghosh D (2007) Efficient characterization of the random eigenvalue problem in a polynomial chaos decomposition. International Journal for Numerical Methods in Engineering 72(4):486-504

[11] Panayirci HM, Schuëller GI, (2011) On the Capabilities of the Polynomial Chaos Expansion Method within SFE Analysis-An Overview, Arch. Comput. Method Eng., vol. 18, no. 1, pp. 43-55 
[12] Wiener N (1938) The homogeneous chaos. American Journal of Mathematics 60(4):897-936

[13] Ghanem RG, Spanos P (1991) Stochastic Finite Elements A Spectral Approach. Springer New York

[14] Le Maître OP, Knio OM (2010) Spectral Methods for Uncertainty Quantification. Springer Netherlands

[15] Ghosh D, Ghanem RG (2008) Stochastic convergence acceleration through basis enrichment of polynomial chaos expansions. International Journal for Numerical Methods in Engineering 73(2):162-184

[16] Ghosh D, Ghanem RG (2012) An invariant subspace-based approach to the random eigenvalue problem of systems with clustered spectrum. International Journal for Numerical Methods in Engineering 91(4):378396

[17] Verhoosel CV, Gutierrez MA, Hulshoff SJ (2006) Iterative solution of the random eigenvalue problem with application to spectral stochastic finite element systems. International Journal for Numerical Methods in Engineering 68(4):401-424

[18] Xiu D, Karniadakis G (2002) The Wiener-Askey polynomial chaos for stochastic differential equations. SIAM J Sci Comput 24(2):619-644

[19] Ernst O, Mugler A, Starkloff H, Ullmann E (2012) On the convergence of generalized polynomial chaos expansions. ESAIM Mathematical Modelling and Numerical Analysis 46:317-339

[20] Press WH, Flannery BP, Teukolsky SA, Vetterling WT (1992) Numerical Recipes in C: The Art of Scientific Computing, Cambridge University Press

[21] Géradin M, Rixen D (1997) Mechanical vibrations: Theory and applications to structural dynamics. J. Wiley \& Sons Ltd

[22] Arbenz P (2012), Lecture notes on solving large scale eigenvalue problems. ETH Zurich. pp. 111

[23] Irvine HM (1981) Cable Structures. MIT Press 